\title{
High-Frequency Néel Relaxation Response for Submillimeter Magnetic Particle Imaging Under Low Field Gradient
}

\author{
Suko Bagus Trisnanto ${ }^{*}$ and Yasushi Takemura $\odot^{\dagger}$ \\ Department of Electrical and Computer Engineering, Yokohama National University, Yokohama, 240-8501, Japan
}

(Received 7 July 2020; revised 30 September 2020; accepted 2 December 2020; published 23 December 2020)

\begin{abstract}
Modulating the relaxation response of magnetic nanoparticles at two distinctive frequencies can be a potential scenario to increase the spatial resolution of magnetic particle imaging (MPI), which is beneficial for biomedical research applications. Comparatively, standard MPI requires a high field gradient to acquire a sharp point-spread function to enable submillimeter imaging of magnetic nanotracers distributed within cells or small-animal models. However, this strategy may face issues in sample handling and signal processing, while decreasing the field gradient reduces the spatial resolution and causes a blurry image. To improve the MPI image quality, we introduce a high-frequency excitation field to temporally encode the Lissajous field-free-point trajectory coupled with a low-frequency magnetization response under field gradient. Unlike regular MPI, our method further decodes the moment relaxation response at narrow sidebands around the excitation frequency to obtain the spatial coordinates of magnetic nanoparticles within a field of view, instead of the magnetization harmonics induced by the driving field. Therefore, the fieldfree-point steering frequencies and the resulting trajectory density are critical parameters to adjust the image resolution. Furthermore, a high signal-to-noise ratio can be achieved by use of a 1-MHz excitation field even with a low amplitude. We also demonstrate two-dimensional image reconstruction of a 1-mm ring-shaped solid Resovist ${ }^{\circledR}$ sample with $0.05-\mathrm{mm}$ thickness, as well as other circular and rod phantoms, placed in a $1.4 \times 1.4 \mathrm{~mm}^{2}$ field of view under $2 \mathrm{~T} / \mathrm{m}$ symmetrical field gradient on the $x-y$ plane and $4 \mathrm{~T} / \mathrm{m}$ on the $z$ axis. Although the spatial resolution achieved appears low to differentiate two-neighboring circular phantoms of dense liquid samples, phase shifting can be further used to resolve image distortion due to the low-frequency relaxation effects.
\end{abstract}

DOI: 10.1103/PhysRevApplied.14.064065

\section{INTRODUCTION}

Magnetic particle imaging (MPI) is an imaging technique that locates and quantifies magnetic nanoparticles by inducing ac magnetic fields under dc field gradient [1]. Being a noninvasive technique, MPI is sensitive only to magnetic nanoparticles as tracers, thus offering high selectivity and contrast particularly for preclinical imaging of small-animal models without background contribution [2]. Owing to superparamagnetism [3], magnetic nanoparticles exhibit nonlinear dynamic magnetization under ac magnetic field, in which the resulting harmonic components and their magnitudes can be significantly reduced by applying a strong dc magnetic field [4]. Standard MPI relies principally on larger magnetization harmonics from

*suko-trisnanto-zt@ynu.ac.jp

†takemura-yasushi-nx@ynu.ac.jp

Published by the American Physical Society under the terms of the Creative Commons Attribution 4.0 International license. Further distribution of this work must maintain attribution to the author(s) and the published article's title, journal citation, and DOI. the particles at the field-free point (FFP) than those from outside the FFP to visualize the spatial distribution of the particles while the FFP or the phantom is moved within the field of view (FOV) [5]. Furthermore, MPI offers high temporal resolution due to a quick scan of the FFP trajectory, which is able to produce more than 46 frames/s [6]. The short signal acquisition time and fast image reconstruction promote MPI as a candidate for real-time imaging of perfusion in the beating heart and the ischemic brain [6-8].

Over a decade, MPI has evolved into several instrumentation setups in order to improve image quality. Wideband MPI is the most-conventional MPI using a single excitation field (i.e., driving field) to induce harmonic responses of the tracers [1]. The intermodulation technique further enriches the harmonic components by superimposing excitation fields with different frequencies [9]. MPI shows its potential as a multicolor imaging method that discriminates the tracers from either their size-dependent relaxation frequencies or harmonic responses [10,11]. In terms of image reconstruction time, the temporal resolution can be increased by magnetically steering the FFP trajectory [12], instead of mechanically moving the sample [1]. Since the magnetostimulation threshold limits the rapidly scanned 
volume [13], a focus field is later introduced to obtain multiple subvolumes that can be combined into large coverage of the FOV $[14,15]$. Here, the static field gradient is critical to define the scan parameters, including excitation frequency and amplitude. Traveling-wave MPI offers more a flexible setup, where a dynamic linear gradient array is introduced to generate and move the FFP simultaneously, instead of using permanent magnets [16]. Moreover, research on the field-free region (e.g., point, line) and its trajectory pattern also contribute to optimizing the image reconstruction $[17,18]$.

MPI promises excellent performance in biomedical applications such as cancer diagnosis, stem-cell tracking, neuroimaging, and blood-pool vascular imaging (angiography) [19-21]. For those in vivo studies, MPI offers zero signal attenuation against the tracer depth and no background signal, whereas optical imaging often shows a surface-weighted signal. This feature originates from the physical and magnetic properties of the tracers. In MPIguided therapy, for instance, magnetic nanoparticles that can be specifically and nonspecifically targeted to cancer cells act as theranostic agents by tracing, visualizing, and heating the cells $[22,23]$. Because of long-term fate of iron oxide tracers, MPI has enabled the tracking of neural-progenitor-cell grafts implanted on the forebrain of rats for more than 3 months [24]. This long-term tracking is not compatible with positron-emission tomography, which uses radioactive tracers with short half-lives. MPI also relies on the dispersity of magnetic nanoparticles in blood plasma or their encapsulation in red blood cells to image the vasculature during the blood circulation [25]. However, whether the tracers are localized or circulated, MPI does not give us the surrounding morphological background where the tracers exist. Therefore, combining MPI with magnetic resonance imaging, computed tomography, or simple optical microscopy will provide a more-comprehensive and more-informative image.

For more-advanced research applications, MPI can be potentially implemented for bioanalytical cellular imaging to study nanoscale drug uptake and cytotoxicity [26]. To compete with fluorescence microscopy, MPI must acquire fine resolution around the micrometer range [27]. The conventional strategy of increasing the spatial resolution is to optimize the excitation frequency and amplitude to obtain large harmonic responses of the tracers [28]. Nevertheless, this scenario should anticipate side effects such as excessive cell heating and peripheral nerve stimulation $[13,29$, 30]. The use of large magnetic nanoparticles can also be effective to increase the signal-to-noise ratio (SNR), while the accompanying relaxation effects can be reduced by using pulsed excitation [31]. Another effective strategy is to reduce the size of the point-spread function (PSF) by increasing the field gradient. Since a high field gradient reduces MPI signals [4], ultrasensitive magnetic sensors are further required to compensate the SNR [21,32].
Our previous work alternatively proposes a method that modulates the harmonic-rich low-frequency magnetization (induced by the FFP-steering fields) with a high-frequency moment relaxation under $35 \mathrm{~T} / \mathrm{m}$ [33]. However, a high field gradient requires careful sample handling where the tracers experience a large magnetic force to displace them. Therefore, we conduct a more-detailed study of this modulated MPI to improve its practicality and feasibility at a low field gradient.

In this paper, we review the fundamental relaxation behavior of magnetic nanoparticles that later emphasizes the different imaging strategy of the modulated MPI from that of standard MPI. We also introduce our prototype of a two-dimensional (2D) submillimeter MPI scanner with a $2 \mathrm{~T} / \mathrm{m}$ field gradient and preliminarily show a one-dimensional (1D) FFP scan. We further demonstrate the image-reconstruction process while changing the scan parameters, such as the FFP steering frequency, the trajectory density, and the profile of the field gradient. We also investigate how the modulated MPI can replicate the phantom morphology for solid samples, whereas the images of liquid phantoms should be corrected because of the Brownian relaxation effects. Moreover, we highlight a small ratio of the low-to-high excitation frequency to increase the spatial resolution of the modulated MPI to compensate the low-field gradient. Since this scan setup will increase the acquisition time and the amount of recorded data significantly, we explore mainly the characteristics of the modulated MPI at an excitation frequency ratio of $2 \mathrm{kHz} / 1 \mathrm{MHz}$.

\section{RELAXATION CHARACTERISTICS OF MAGNETIC NANOPARTICLES}

In principle, the dynamic magnetization response of magnetic nanoparticles is an important MPI signal that can be spatially differentiated by introducing a static field gradient. This response varies among particles of different chemical compounds, sizes, and structures as they exhibit unique relaxation behavior individually [34-36]. Regarding these physical properties, there are two factors that significantly contribute to the MPI signal, how large the magnetic moments are and how fast the relaxation occurs. Polydispersity makes both the magnetic moment and the relaxation time constant statistically distributive [37]. Nevertheless, we may simply use the mean magnetic moment and effective relaxation time to characterize the MPI signal [38]. If we define $\hat{\mathbf{e}}, \hat{\boldsymbol{\mu}}$, and $\hat{\mathbf{h}}$ as unit vectors of magnetization, moment, and field, respectively, the scalar analysis of the magnetization $\mathbf{M}=$ Mê and magnetic moment $\mathbf{m}=$ $m \hat{\boldsymbol{\mu}}$ as a function of the magnetic field $\mathbf{H}=H \hat{\mathbf{h}}$ can be used to evaluate the statistical properties of magnetic nanoparticles. Therefore, the evaluation of magnetization hysteresis and relaxation spectra is necessary to further understand the relaxation characteristics of magnetic nanoparticles. 


\section{A. Magnetization hysteresis}

Depending on the particle size, magnetic nanoparticles may exhibit superparamagnetism, in which their magnetization is nonlinearly dependent on the field with no remanence in a static field-free environment and saturates with high magnetization, as described by the Langevin function $\mathcal{L}$ [Eq. (1)] [39]. Equation (1) implicitly means that the field-induced relaxation behavior of the particles critically determines the nonlinearity of the magnetization:

$$
M\left(H_{\mathrm{dc}}\right)=\frac{1}{v} \int m_{p} \mathcal{L}\left(\frac{\mu_{0} m_{p} H_{\mathrm{dc}}}{k_{B} T}\right) \rho_{m} d m_{p} .
$$

When suspended in a viscous medium, magnetic nanoparticles can randomly move their hydrodynamic nanostructures to minimize the magnetopotential energy for a given external static field. This situation enhances the nonlinearity of dc magnetization. In contrast, for immobilized nanoparticles, which exhibit only the moment relaxation, the static magnetization response tends to be linear in the low-field regime. From Fig. 1(a), for instance, a liquid sample of Resovist ${ }^{\mathbb{R}}$ (Fujifilm RI Pharma, Japan) shows higher magnetization below $50 \mathrm{mT} / \mu_{0}$ than a solid sample; $\mu_{0}$ is the magnetic permeability of free space. The contribution of Brownian relaxation can be further identified from the change in the magnetic moment distribution.

In the case of superparamagnetism, the nonlinear increase of magnetization $M$ with increasing static field $H_{\mathrm{dc}}$ involves the sum of each composing magnetic moment $m_{p}$ over the sample volume $v$, while following the Langevin function [Eq. (1)] [40,41]. The ratio of magnetic energy $\left(\mu_{0} m_{p} H_{\mathrm{dc}}\right)$ to thermal energy $\left(k_{B} T\right.$, where $k_{B}$ and $T$ are Boltzmann constant and absolute temperature, respectively) acts as the Langevin parameter. Considering the probability density function of magnetic moments $\left(\rho_{m}\right)$ and the saturation magnetization $\left(M_{S}\right), m_{p}$ can be estimated by deriving the inverse Langevin function of the normalized $M$ shown as Eq. (2) [37]:

$$
m_{p}=\frac{k_{B} T}{\mu_{0}} \frac{d \mathcal{L}^{-1}\left(M / M_{S}\right)}{d H_{\mathrm{dc}}} .
$$

Since typical dc magnetometry (e.g., with a vibratingsample magnetometer) provides a set of $M\left(H_{\mathrm{dc}}\right)$ data with a finite $H_{\mathrm{dc}}$ increment, Eqs. (1) and (2) can be further solved in their discrete forms to obtain the probability mass function of magnetic moments $\left(W_{m}\right)$. From the inset in Fig. 1(a), the superparamagnetic $M\left(H_{\mathrm{dc}}\right)$ curves of a commercially available Resovist sample reveal the polydispersity of the suspended maghemite nanoparticles with the broadening magnetic moment distribution.

Figure 1(a) identifies a secondary peak in the magnetic moment distribution of the liquid Resovist sample with a large value attributed to the cluster moment, $\mathbf{m}_{c}=$
$\sum_{i=1}^{I} \mathbf{m}_{p, i}$ [42]. As a semirigid-dipole system [43], I magnetic nanoparticles form hydrodynamic nanoclusters in a liquid medium, where the resultant of all composing particle moments $\mathbf{m}_{p, i}$ is nonzero, thus enabling Brownian relaxation of the secondary volume with angular velocity $\hat{\mathbf{\omega}}_{c}$ in the presence of an external field $\mathbf{H}_{e}$. Here $\mathbf{H}_{e}$ includes not only $\mathbf{H}_{\mathrm{dc}}$ but also fields associated with thermal fluctuation, anisotropy energy, and dipolar magnetism. Equation (3) further defines the dynamics of cluster moments by taking the internal Néel relaxation into account:

$$
\frac{d \mathbf{m}_{c}}{d t}=\sum_{i=1}^{I} \frac{d \mathbf{m}_{p, i}}{d t}+\omega_{c} \times \sum_{i=1}^{I} \mathbf{m}_{p, i} .
$$

In this case, the dipolar interparticle interaction of a single nanocluster may not be sufficiently strong to completely block thermal relaxation of $\mathbf{m}_{p, i}$ [44]. The resulting moment dynamics $d \mathbf{m}_{p, i} / d t$ follows the stochastic LandauLifshitz-Gilbert equation expressed as Eq. (4) to undergo precessional motion:

$$
\frac{d \mathbf{m}_{p, i}}{d t}=\frac{-\gamma}{1+\alpha^{2}}\left[\mathbf{m}_{p, i} \times \mathbf{H}_{e, i}+\alpha \mathbf{m}_{p, i} \times\left(\mathbf{m}_{p, i} \times \mathbf{H}_{e, i}\right)\right],
$$
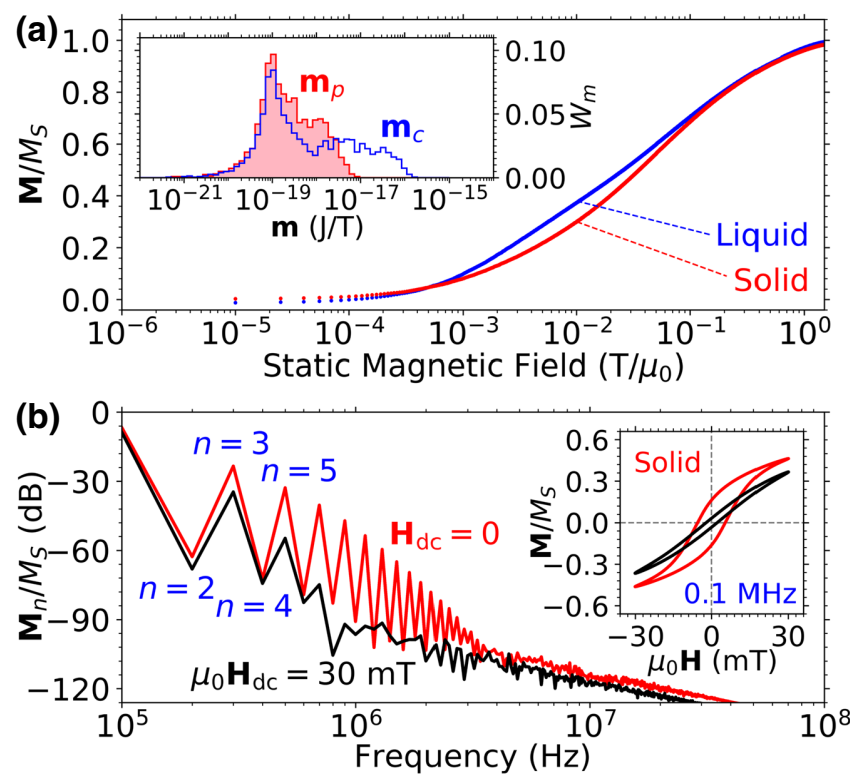

FIG. 1. Magnetization dynamics of Resovist emphasizing (a) superparamagnetic de magnetization curves of liquid and solid samples and (b) ac magnetic field-induced magnetization harmonics of a solid sample. The magnetic moment distribution estimated from the inverse Langevin function of $\mathbf{M} / M_{S}$ reveals large cluster moments $\mathbf{m}_{c}$ in the liquid sample. Under highfrequency ac field, moment relaxation of the core particles results in magnetization hysteresis that is sensitive to the presence of the static field. The corresponding harmonic magnitude $\mathbf{M}_{n} / M_{S}$ can be further obtained by Fourier transform of the dynamic $\mathbf{M} / M_{S}$. 
where $\alpha$ is the Gilbert damping constant and $\gamma$ is the gyromagnetic ratio. The ideal rigid-dipole system has $d \mathbf{m}_{p, i} / d t=0$ due to the thermally blocked internal moment dynamics [45].

The Néel relaxation depends strongly on the particle size, where large size results in large anisotropy energy as well as a long Néel time constant [46]. Thermal moment relaxation is insignificant for large magnetic nanoparticles, thus satisfying the rigid-dipole model. In minimizing the relaxation effects, MPI prefers magnetic nanoparticles within the monodomain size limit [47-49]. The existence of Néel relaxation can be further observed in the dynamic magnetization of a solid sample (i.e., immobilized nanoparticles) at high frequency. Figure 1(b) gives an example of the dynamic response of a solid Resovist sample at $100 \mathrm{kHz}$. We can extract the corresponding magnetization harmonics $M_{n}$ from the hysteresis loop [Fig. 1(b) inset] through Fourier transform, in which the hysteresis area itself is related to the relaxation losses [50].

A sinusoidal magnetic field with sufficiently large amplitude generates odd harmonic components of the magnetization response, which corresponds to a Taylor expansion of the Langevin function [5]. From Fig. 1(b), applying $\mathbf{H}_{\mathrm{dc}}$ perpendicular to the ac field can reduce the harmonic magnitudes, particularly for high orders of $n \geq 5$. Standard MPI uses these static field-dependent harmonic responses to distinguish the signal coming from the FFP. From Fig. 1(b), the presence of a static field reduces and linearizes the magnetic response due to an increase in the relaxation frequency [51]. However, the magnetic response at the fundamental frequency appears less field dependent than the magnetic responses at the harmonic frequencies, emphasizing that Néel relaxation can survive under high dc bias field. The modulated MPI exploits this characteristic to further modulate the low-frequency magnetization response induced by the FFP-steering fields [33]. This strategy relies on magnetic responses at two distinctive excitation frequencies, thus requiring an understanding of the magnetic susceptibility spectra of magnetic nanoparticles to optimize the MPI signal.

\section{B. Relaxation spectra}

The relaxation behavior of a single magnetic nanoparticle ideally follows the Debye model, which highlights the first-order differential equation of the magnetization response with a characteristic relaxation time constant $\tau$ [52]. Equation (5) further describes $M_{\infty}$ and $M_{i}$ as empirical high-frequency and low-frequency limiting magnetizations, respectively:

$$
\tau \frac{d M(t)}{d t}+M(t)=\tau \frac{d M_{\infty}(t)}{d t}+M_{i}(t) .
$$

For an ideal noninteracting uniform nanoparticle system, $M_{i}$ is equivalent to $\chi_{0} H$, where $\chi_{0}$ is the equilibrium susceptibility obtained under quasistatic field, whereas $M_{\infty}$ is often negligible. However, a polydispersive nanoparticle system empirically has nonzero $M_{\infty}$ due to inhomogeneous relaxation responses [53]. $M_{\infty}=\chi_{\infty} H$ is later attributed to the intrapotential-well contribution with $\chi_{\infty}=\left(\mu_{0} \in M_{S}^{2}\right) / K_{a}$, where $\epsilon$ and $K_{a}$ are the volume fraction and effective anisotropy constant of the particles, respectively [54]. Equation (6) generalizes the frequencydomain steady-state magnetization response of a polydispersive nanoparticle system by introducing the probability density function of the relaxation time $\left(\rho_{\tau}\right)$ :

$$
M(\omega)=\int\left(M_{\infty}+\frac{M_{i}-M_{\infty}}{1+i \omega \tau}\right) \rho_{\tau} d \tau
$$

Equation (6) also describes the complex magnetization for a given sinusoidal field $H(t)=H_{0} \cos \omega t$ with amplitude $H_{0}$ and frequency $f=\omega / 2 \pi$, which is typically expressed in terms of real $\chi^{\prime}$ and imaginary $\chi^{\prime \prime}$ parts of the magnetic susceptibility as $M(\omega)=\left[\chi^{\prime}(\omega)-i \chi^{\prime \prime}(\omega)\right] H_{0}$.

Magnetic nanoparticles suspended in a liquid medium have two characteristic time constants, which are attributed to the physical particle rotation and magnetic moment rotation. From Eq. (3), the coupled Brownian and Néel relaxations follow linear-response theory, and thus it is necessary to use a low field strength (e.g., typically less than $0.5 \mathrm{mT} / \mu_{0}$ ) to induce a linear magnetization response associated with intrinsic relaxation behavior [51]. A high field amplitude not only results in nonlinearity of the magnetic response but also increasing relaxation frequency. To analyze the Brownian relaxation time $\tau_{B}$, for example, Fig. 2(a) shows the frequency-dependent complex magnetic susceptibility of a commercial ferrofluid (M300, Sigma Hi-Chemical, Japan), for which the viscosity has been increased to shift the Brownian relaxation frequency to $554 \mathrm{~Hz}$. Here $\chi^{\prime}$ and $\chi^{\prime \prime}$ are obtained by spectroscopic magnetometry with a finite logarithmic frequency increment and $H_{0}=0.1 \pm 0.03 \mathrm{mT} / \mu_{0}$.

From Fig. 2(a), the liquid M300 sample appears to have larger $\chi^{\prime}$ at $f \leq\left(2 \pi \tau_{B}\right)^{-1}$ than the solid sample. For $f \gg\left(2 \pi \tau_{B}\right)^{-1}, \chi^{\prime}$ further decreases since the suspended particles experiencing a large rotational friction force at high frequency tend to physically relax their easy axes across the applied field direction with a large phase delay $\varphi=\tan ^{-1}\left(\chi^{\prime \prime} / \chi^{\prime}\right)$ relative to $H(t)$. The Brownian easyaxis alignment is also responsible for slightly smaller $\chi^{\prime}$ in the liquid sample (than in the solid sample) at high frequency, where the resulting magnetization vector is less parallel to the applied field [55]; the solid sample contains nanoparticles with randomly oriented easy axes. Nevertheless, the high-frequency easy-axis relaxation behavior may be more complex for $H_{0} \gg 0.1 \mathrm{mT} / \mu_{0}$ overcoming the anisotropy field of the particles [56]. In this case, the easy 
(a)

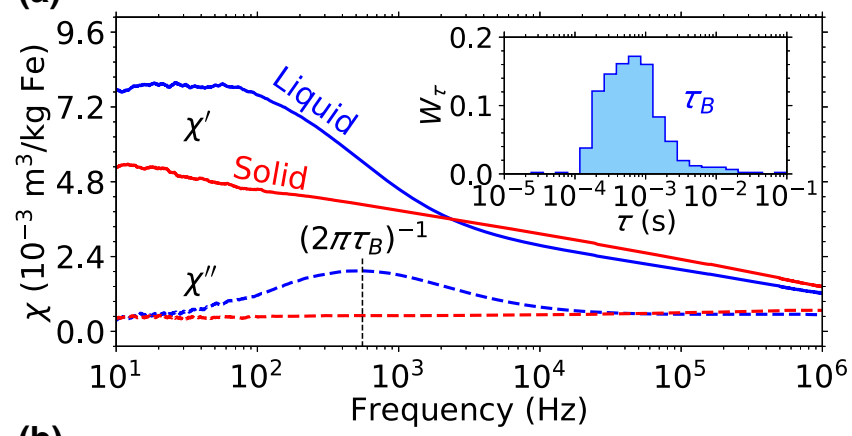

(b)

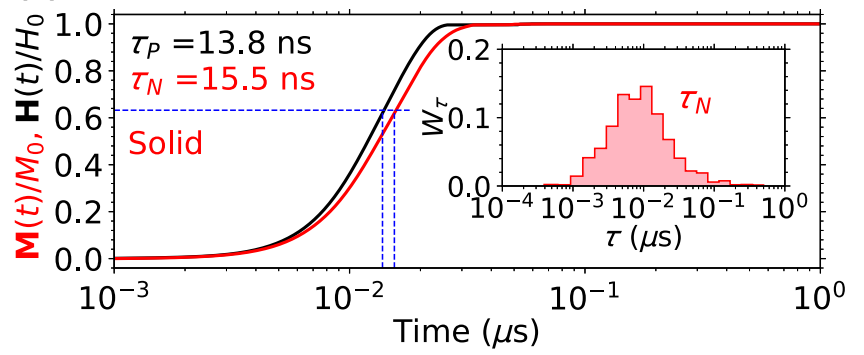

FIG. 2. Steady-state and transient magnetization responses of a viscosity-adjusted M300 ferrofluid in terms of (a) frequencydependent complex magnetic susceptibility at $0.1 \mathrm{mT} / \mu_{0}$ and (b) magnetization response of a solid sample under $0.5 \mathrm{mT} / \mu_{0}$ pulsed excitation. The imaginary part of the magnetic susceptibility $\chi^{\prime \prime}$ identifies the Brownian peak in the liquid sample, which is further attributed to the distribution of the Brownian relaxation time $\tau_{B}$. The pulsed field induces a slightly delayed magnetization response in the solid sample, in which the time-constant distributions of Néel relaxation $\left(\tau_{N}\right)$ and the pulse $\left(\tau_{p}\right)$ should partially overlap.

axes more parallel to the applied field may lead to larger magnetization than those randomly oriented. The probability mass function $\left(W_{\tau}\right)$ of the intrinsic Brownian relaxation time can be later estimated from $\tau_{B}=d\left[\omega^{-1} \chi^{\prime \prime}\left(\chi^{\prime}\right)\right] / d\left(\chi^{\prime}\right)$ to satisfy Eq. (6) in a discrete form [Fig. 2(a), inset] [37].

The moment dynamics of superparamagnetic nanoparticles occurs faster than the Brownian relaxation of the corresponding hydrodynamic structures [43]. Figure 2 confirms that $\chi^{\prime \prime}$ of the solid sample shows no observable peak at a frequency up to $1 \mathrm{MHz}$. To further evaluate Néel relaxation spectra, transient magnetometry is a favorable method and uses a pulsed excitation field $H(t)=H_{0} u(t)$ to instantly magnetize the particles. From Eq. (5), the magnetization response is given by $M(t)=M_{0}\left[1-\exp \left(-t / \tau_{N}\right)\right]$, with $M_{0}$ the steady-state magnetization, and thus the Néel relaxation time constant $\tau_{N}$ can be estimated from $\tau_{N}=d\left[1-M(t) / M_{0}\right] / d \dot{M}(t)$. However, the excitation field is practically defined as $H(t)=H_{0}\left[1-\exp \left(-t / \tau_{p}\right)\right]$ with short pulse time constant $\tau_{p}<\tau_{N}$. Therefore, $M(t)$ also includes the magnetization response at frequency $f=\left(2 \pi \tau_{p}\right)^{-1}$. Figure 2(b) shows the delayed magnetization response of the solid M300 sample relative to the $0.5 \mathrm{mT} / \mu_{0}$ pulsed field, emphasizing the distributive probability mass function of $\tau_{N}$. The effective $\tau_{N}(15.5 \mathrm{~ns})$ is significantly smaller than the effective $\tau_{B}(0.3 \mathrm{~ms})$. The modulated MPI principally uses distinctive relaxation timescales to exploit the magnetization responses associated with the Brownian and Néel relaxations.

\section{SUBMILLIMETER MAGNETIC PARTICLE SCANNER}

An MPI system typically consists of permanent magnets generating a static field gradient, pairs of coils producing fields which steer the FFP trajectory, focus the FOV, and drive the harmonic responses, and gradiometric receive coils. Here the modulated MPI scanner uses two sets of permanent magnets to produce a $2 \mathrm{~T} / \mathrm{m}$ symmetrical field gradient on the $x$ and $y$ axes, while the $z$ axis experiences $4 \mathrm{~T} / \mathrm{m}$ (Fig. 3 and Appendix A); the usual field gradient for MPI can be less than $2 \mathrm{~T} / \mathrm{m}$. For $\hat{\mathbf{i}}_{x}, \hat{\mathbf{i}}_{y}$, and $\hat{\mathbf{i}}_{z}$ as the axial unit vectors, the resulting FFP is then steered by two perpendicular oscillatory fields, $\mathbf{H}_{x}=H_{x} \hat{\mathbf{i}}_{x}$ and $\mathbf{H}_{y}=H_{y} \hat{\mathbf{i}}_{y}$, with identical frequencies at $f_{x}$ and $f_{y}$, to move in a dense Lissajous trajectory. An additional high-frequency $z$-axis field $\left(\mathbf{H}_{z}=H_{z} \hat{\mathbf{i}}_{z}\right)$ is applied at frequency $f_{z}=1 \mathrm{MHz}$ to induce the Néel relaxation of the tracers. Equation (7) shows that the relaxation modulation temporally encodes the magnetization response at the moving FFP $\left[M_{x}(t)\right.$ and $\left.M_{y}(t)\right]$ as a distorted $z$-axis magnetization, $\mathbf{M}_{z}^{*}=M_{z}^{*} \hat{\mathbf{i}}_{z}$ :

$$
M_{z}^{*}(t)=\left[1+a_{x} M_{x}(t)+a_{y} M_{y}(t)\right] M_{z}(t) .
$$

where $a_{x}$ and $a_{y}$ are the amplitude sensitivities for $M_{x}(t)$ and $M_{y}(t)$, respectively [33]. We use a sampling rate of 12.5 Megasamples per second (MS/s) to capture the $M_{z}^{*}$ signal. From Eq. (8), the $z$-axis magnetization $\left(\mathbf{M}_{z}=M_{z} \hat{\mathbf{i}}_{z}\right)$ itself depends on the equilibrium susceptibility $\left(\chi_{z}\right)$ of the tracers and their relaxation time distribution $\left(\rho_{\tau}\right)$ at angular frequency $\omega_{z}=2 \pi f_{z}$ for a given $H_{z}$ and $G_{z} z$ fields; $G_{z}$ is the field gradient on the $z$ axis:

$$
M_{z}(z, t)=\chi_{z}\left[\frac{G_{z} z}{\mu_{0}}+\int\left(H_{z}-\tau \frac{\partial H_{z}}{\partial t}\right) \frac{\rho_{\tau} d \tau}{1+\left(\omega_{z} \tau\right)^{2}}\right] .
$$

The low-frequency magnetizations on the $x$ and $y$ axes, $\mathbf{M}_{x}=M_{x} \hat{\mathbf{i}}_{x}$ and $\mathbf{M}_{y}=M_{y} \hat{\mathbf{i}_{y}}$, must also satisfy Eq. (8), while the Brownian particle dynamics contributes to the effective relaxation time $\tau \approx \tau_{B}$ for the liquid sample.

To evaluate the modulated MPI scanner, maghemite nanoparticles of Resovist ( $28 \mathrm{mg}$ Fe per milliliter) contained in capillary glass tubes with inner diameters of $0.4,0.6$, and $1 \mathrm{~mm}$ are used as the phantoms. With pulsating $\mathbf{H}_{x} \perp \mathbf{H}_{y} \perp \mathbf{H}_{z}$ fields at a 10-s interval with a $0.5 \%$ duty cycle and a ratio of the field amplitude 

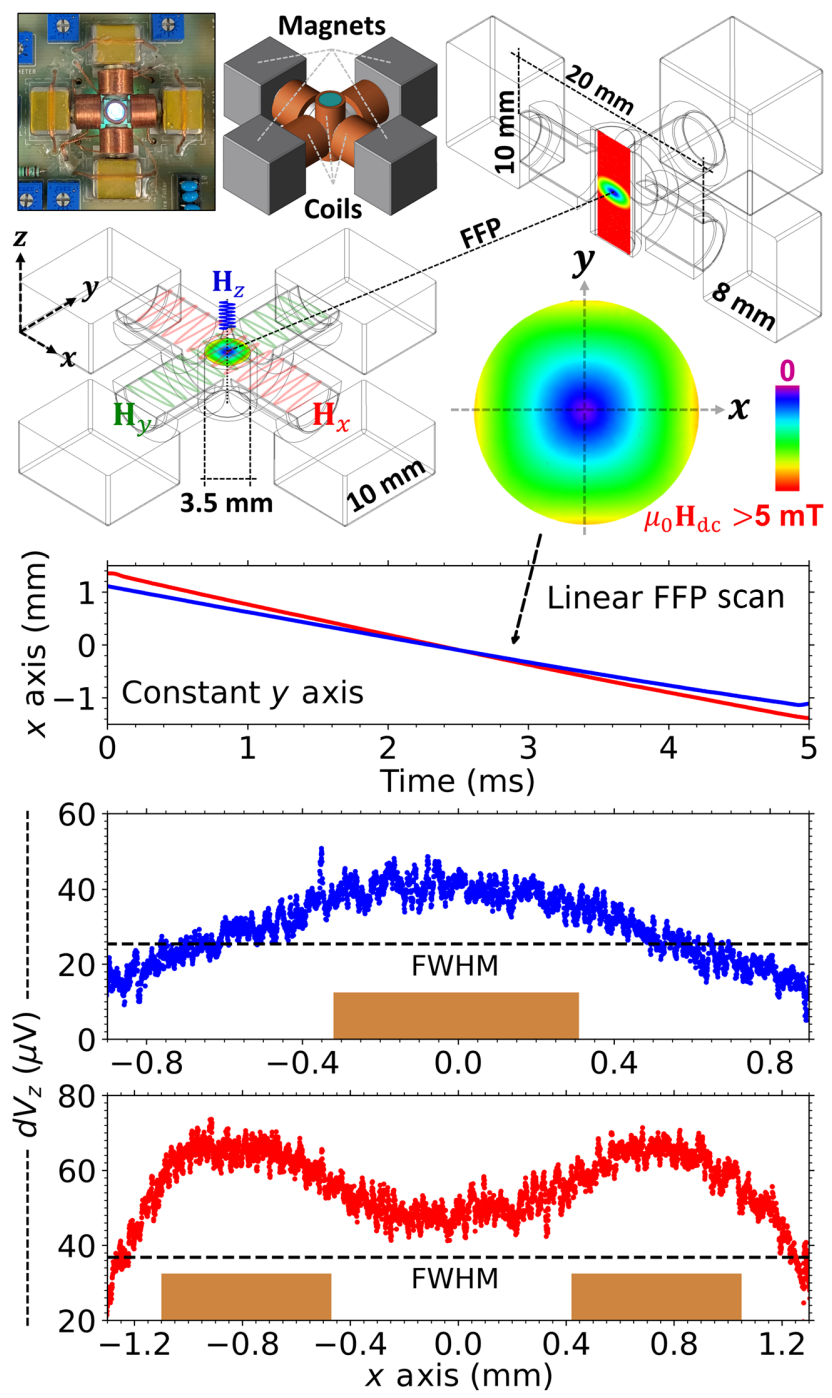

FIG. 3. MPI scanner design and 1D magnetic particle spectroscopy. Four magnets facing the same polarity create a FFP with field gradient $G_{x}=G_{y}=2 \mathrm{~T} / \mathrm{m}$ and $G_{z}=4 \mathrm{~T} / \mathrm{m}$, simulated by JMAG-Designer 19.0. The effective $2 \mathrm{D}$ FOV is $1.4 \times$ $1.4 \mathrm{~mm}^{2}$ within a $3.5-\mathrm{mm}$ bore. The extended low-frequency $x$-axis FFP scan of liquid Resovist phantoms further confirms the spatial resolution of the scanner.

$H_{x, 0}: H_{y, 0}: H_{z, 0}=10: 10: 1, M_{z}^{*}(t)$ of the tracers is measured as an electromotive force $V_{z}(t)$ [Eq. (9)] with use of a gradiometric receive coil with geometric sensitivity $K$ [57]:

$$
V_{z}(t)=-\mu_{0} K \iiint \frac{\partial M_{z}^{*}(t)}{\partial t} d x d y d z
$$

Before the data averaging, $V_{z}(t)$ further passes through a 1$\mathrm{MHz}$ bandpass filter to extract its time-varying amplitude at a narrow bandwidth $f_{z} \pm n f_{x, y}$ relative to the noise fluctuation (i.e., signal demodulation). $d V_{z}(t)$ is later obtained from the half-rectified $V_{z}(t)$ signal through synchronous envelope tracking at $1 / f_{z}$. The spatial decoding of the signal coordinate within the sample into a 2D image is based on the temporally equidistant signal mapping on the predefined Lissajous trajectory of the FFP, further followed by regridding and spatial Gaussian filtering [33]. The image slices obtained by mechanically sliding the sample can be stacked to acquire a three-dimensional (3D) image. In this section, we explain and demonstrate the MPI scanner performance in reconstructing $1 \mathrm{D}, 2 \mathrm{D}$, and $3 \mathrm{D}$ images.

\section{A. One-dimensional FFP scan}

The spatial resolution is the most crucial specification of a MPI scanner, and represents the scanner's capability to differentiate two neighboring phantoms. To evaluate this parameter, we perform linear FFP scans of the liquid Resovist samples along the $x$ axis at two different scan speeds: 0.18 and $0.52 \mathrm{~ms}^{-1}$. Since the bore size is 3.5 $\mathrm{mm}$, the 1D FFP scan can be extended up to $\pm 1.3 \mathrm{~mm}$ by adjustment of $H_{x}$. Two identical samples are prepared by our filling $0.6-\mathrm{mm}$-inner-diameter capillary tubes with $0.21 \mu 1$ Resovist; the mass of each sample is approximately $5.9 \mu \mathrm{g}$ Fe. Figure 3 further shows a $0.18-\mathrm{ms}^{-1} 1 \mathrm{D}$ FFP scan of a single circular phantom and a $0.52-\mathrm{ms}^{-1}$ 1D FFP scan of double circular phantoms for $5 \mathrm{~ms}$. The full width at half maximum (FWHM) of the demodulated signal amplitude $\left(d V_{z}\right)$ identifies the diameter of the phantoms. Moreover, the FWHM signal can distinguish the double circular phantoms (surface-to-surface) distanced by $0.9 \mathrm{~mm}$. From Fig. 3, the spatial resolution should be less than $1.5 \mathrm{~mm}$ (center-to-center distance of the phantoms). See Appendix B for more details about the evaluation of spatial resolution. Nevertheless, the 1D FFP scan is not sufficiently informative to observe the Brownian relaxation effects in the liquid phantom.

\section{B. Two-dimensional image reconstruction}

To obtain the 2D image, the FFP movement is necessarily steered by $H_{x}(t)$ and $H_{y}(t)$ to enable the Lissajous trajectory $y(x)$, where the corresponding $x$ and $y$ axial FFP movements are defined as $x(t)=H_{x}(t) / G_{x}$ and $y(t)=H_{y}(t) / G_{y}$, respectively. As the phantom for demonstrating the image reconstruction process, $0.16 \mu \mathrm{l}$ Resovist is put inside a capillary tube with 1-mm inner diameter, then evaporated to form a $4.4 \mu \mathrm{g}$ Fe solid ring phantom with $0.05-\mathrm{mm}$ thickness. As shown in Fig. 4, $V_{z}(t)$ generated by the ring phantom reference has a high SNR since $V_{z}(t)$ is proportional to $\dot{M}_{z}^{*}(t)$ [Eq. (9)]. Here $V_{z}(t)$ may further disregard the differential component $\left[a_{x} \dot{M}_{x}(t)+a_{y} \dot{M}_{y}(t)\right] M_{z}(t)$ due to $M_{z}(t) \ll \dot{M}_{z}(t)$, thus suggesting Eq. (10):

$$
V_{z}(t) \approx-\mu_{0} K v\left[1+a_{x} M_{x}(t)+a_{y} M_{y}(t)\right] \dot{M}_{z}(t)
$$

High-frequency $M_{z}$ is responsible for signal amplification of $V_{z}$ by $\omega_{z}$ relative to the noise background (sample-free generated signal). Figure 4 also confirms that the amplitude modulation $d V_{z}(t)$ has frequency sidebands $f_{z} \pm n f_{x y}$, 
(a)
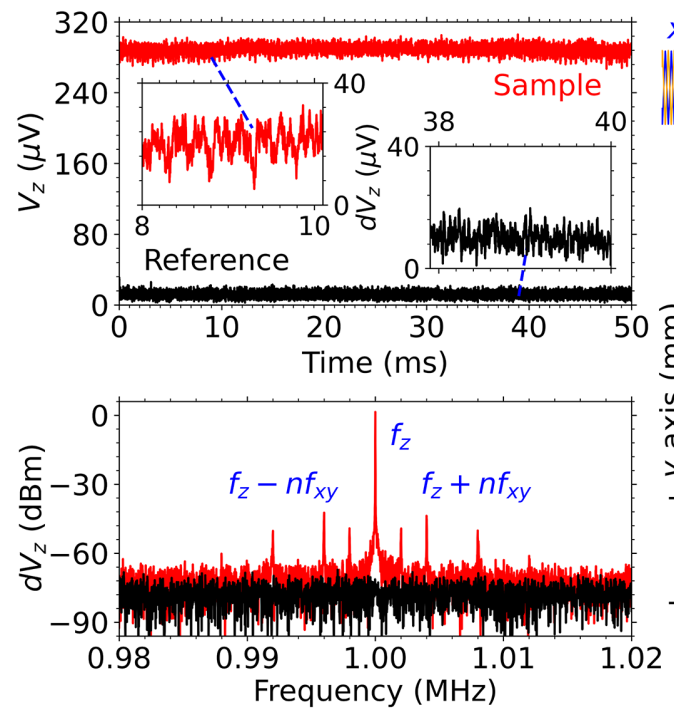

(b)

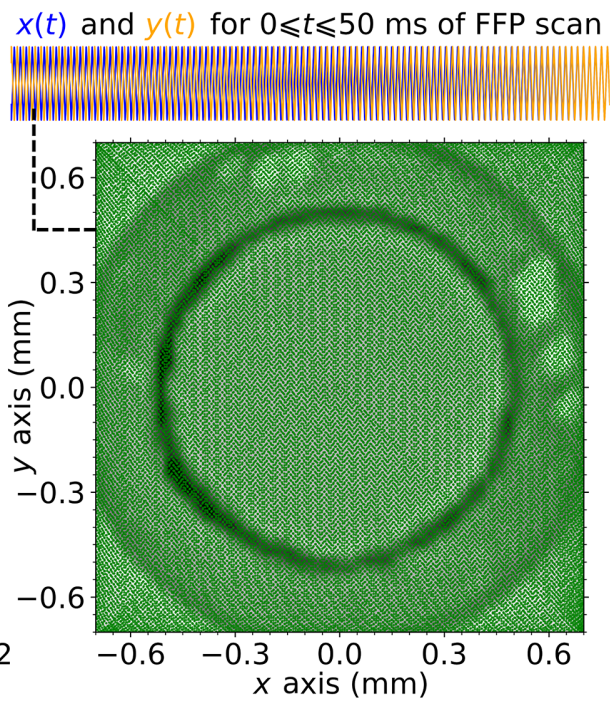

(c)

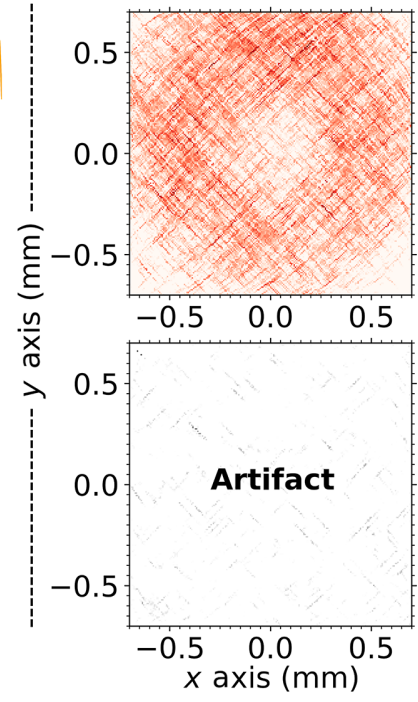

FIG. 4. (a) Modulated signal characteristics, (b) Lissajous-FFP-trajectory coverage, and (c) direct signal decoding of ring-shaped solid Resovist sample at $f_{x}=2 \mathrm{kHz}, f_{y}=2.01 \mathrm{kHz}$, and $f_{z}=1 \mathrm{MHz}$. For a 50 -ms signal acquisition time, the sample-generated voltage $\left(V_{z}\right)$ relative to that of the sample-free reference has a high SNR and shows an amplitude modulation $\left(d V_{z}\right)$. The spectrum characteristic further shows the frequency sidebands around $f_{z}$, which represents the magnetization response during the FFP movement on the $x$ and $y$ axes. The instant coordinates of the FFP at $x(t)$ and $y(t)$ show a Lissajous curve covering the phantom. The temporally equidistant mapping of $d V_{z}(t)$ at the FWHM on the dense Lissajous FFP trajectory as $d V_{z}(x, y)$ enables 2D sample imaging, where the sample-free reference produces an artifact.

which can be attributed to the magnetization response due to the time-varying FFP in terms of $a_{x} M_{x}(t)+a_{y} M_{y}(t)$.

To decode $d V_{z}(t)$ into spatial coordinates of the tracers, $d V_{z}(t)$ can be directly plotted against the time-varying FFP coordinate for $x(t)$ and $y(t)$ with an equidistant time approach as $d V_{z}(x, y)$. From Fig. $4, d V_{z}(x, y)$ shows the 2D image of the phantom, compared with the sample-free $d V_{z}(x, y)$ resulting in an artifact. In this modulated MPI, the time-domain image reconstruction involves the mean magnetic moment for $\dot{M}(x, y, z, t)=c(x, y, z) \dot{m}(x, y, z, t)$ with spatial distribution of the tracer concentration $c$. From Eq. (10), our image-reconstruction method is principally to correlate $c(x, y)$ with $d V_{z}(x, y)$ since $v \dot{M}_{z}(t)=\dot{m}(x, y, z, t)$. Here a dense Lissajous trajectory covering the phantom is crucial to enable direct signal mapping on the $x-y$ plane. The Lissajous trajectory density $N_{D}$ itself can be defined as $f_{x} /\left(f_{y}-f_{x}\right)$. We later discuss the effect of changing $f_{x}$ and $N_{D}$ on the reconstructed image. Image-processing protocols (e.g., regridding, filtering) can be further applied to $d V_{z}(x, y)$ to improve the visualization of the phantom.

\section{Three-dimensional tomography}

MPI technology can be implemented as an alternative for tomographic imaging, in which 3D imaging of the tracers can be typically achieved by installing the drive-field, selection-field, and receive coils on the $x, y$, and $z$ axes [6]. In the case of a 2D MPI scanner, 3D visualization of the tracers can be achieved by stacking each $x-y$ image slice taken at different $z$-axis positions of the sample. Figure 5 shows a 3D tomographic image of two solid phantoms made from $0.09 \mu 1$ Resovist which is put inside a capillary tube with 0.4-mm inner diameter. These circular phantoms are surface-to-surface distanced by $0.5 \mathrm{~mm}$ on both the $x$ axis and the $z$ axis. They are mechanically moved along the $z$ axis by a micrometer slider. Seven image slices are taken with a $0.2-\mathrm{mm}$ interval. From Fig. 5, slice $a$ and slice $g$ clearly confirm the actual position of each phantom. Since the field gradient should change along the $z$ axis, mechanical positioning of the sample on the scanned $x-y$ plane is less effective than using 3D selection fields. Our method relies on the magnetization response due to the modulated field gradient, and thus the spatial resolution may be reduced when the sample is not well positioned in the FPP trajectory. We later discuss the profile of the field gradient affecting the spatial resolution.

\section{EXPERIMENT}

Figures 3-5 demonstrate the preliminary evaluation of the submillimeter modulated MPI scanner in the 1D, 2D, and 3D perspectives. To improve the practicality and feasibility of the modulated MPI for submillimeter imaging, we focus mainly on optimizing the scan parameters to increase the spatial resolution of a $2 \mathrm{D}$ image. Here we 


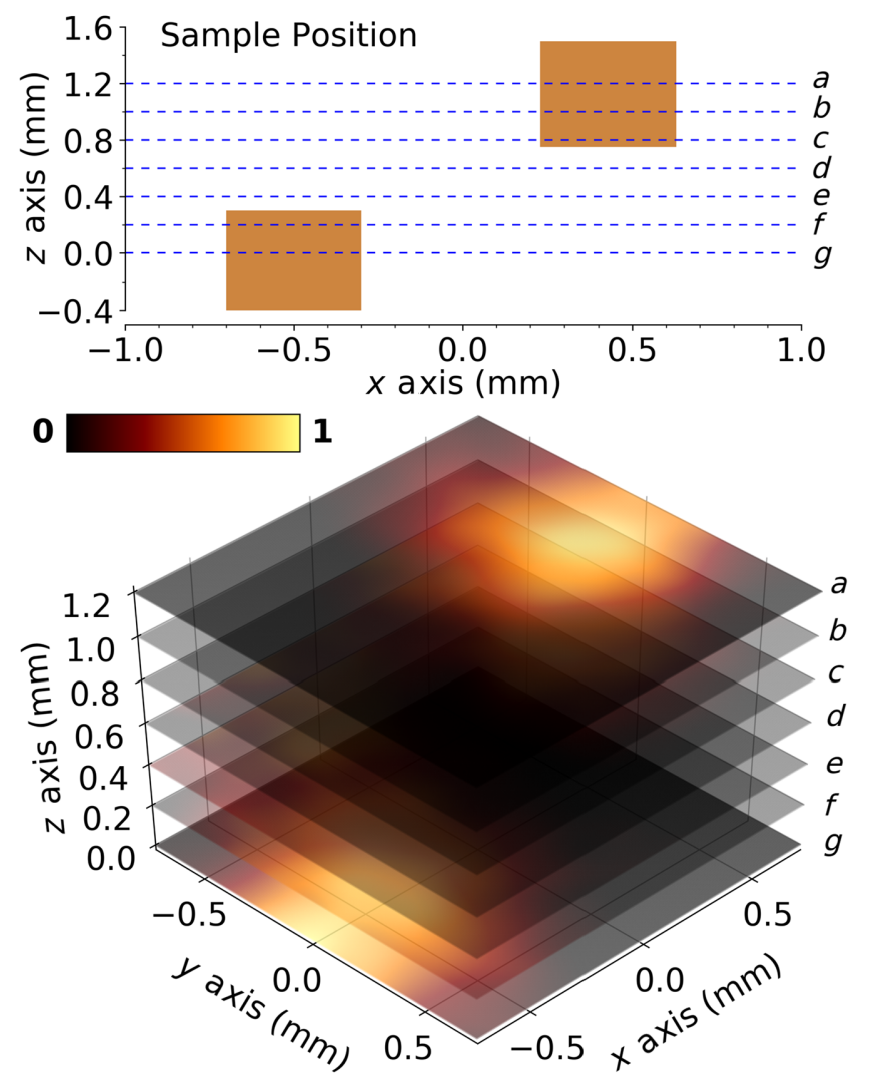

FIG. 5. Three-dimensional tomograpic imaging of two 0.4-mm circular solid phantoms made from Resovist. While the phantoms are center-to-center distanced $0.9 \mathrm{~mm}$ horizontally and $12 \mathrm{~mm}$ vertically $(a-g)$, image slices are taken and stacked to obtain a $3 \mathrm{D}$ image. Each slice was obtained for $f_{x}=2 \mathrm{kHz}$, $N_{D}=200$ and $72.5 \times 72.5 \mu \mathrm{m}^{2}$ Gaussian filter.

use a solid Resovist ring phantom and scan it for various $f_{x}$ (e.g., $0.5,1,2$, and $4 \mathrm{kHz}$ at constant $N_{D}=200$ ), $N_{D}$ (e.g., 50, 100, 200, 300, 400, and 500 at constant $f_{x}=2 \mathrm{kHz}$ ), and $G_{z}$ (e.g., $1.3,1.5$, and $2 \mathrm{~T} / \mathrm{m}$ at constant $f_{x}=2 \mathrm{kHz}$ and $\left.N_{D}=200\right)$ by using the designed MPI scanner. Both $f_{x}$ and $N_{D}$ define the record length $\left(t_{R L}\right)$, where $f_{x}=2 \mathrm{kHz}$ and $N_{D}=200$ result in $t_{R L}=50 \mathrm{~ms}$. In the case of field-gradient dependence, additional FFP simulation is done with JMAG-Designer 19.0 (JSOL Corporation, Japan) to further support the discussion. We also evaluate the solid-ring and cylinder phantoms at different positions and orientations to investigate the scanner precision and accuracy. Moreover, we compare solid and liquid phantoms to show the Brownian relaxation effects and introduce phase shifting to correct the reconstructed image. Spectroscopic magnetometry is additionally used to measure the phase delay of the magnetization response relative to the applied field for both solid and liquid Resovist samples. The imaging is performed at $f_{z}=1 \mathrm{MHz}$ and $H_{z, 0} \approx 0.15 \mathrm{mT} / \mu_{0}$ pulsated at 10 -s intervals, and all the images obtained are Gaussian filtered at an effective area of $55 \times 55 \mu \mathrm{m}^{2}$ and normalized relative to each maximum $d V_{z}(x, y)$.

\section{A. Instrumentation setup}

Principally, the modulated MPI has several fundamental differences in comparison with (standard) narrowband MPI in terms of the excitation field, magnetic signals, and image reconstruction. While narrowband MPI uses a twotone excitation field $\mathbf{H}_{e}$ at high $f_{0}$ and low $f_{1}$ and reads the resulting magnetization harmonics at $m f_{0} \pm(n-1) f_{1}$ (for $m, n=2,3,4, \ldots$ ) [9], the modulated MPI simply uses a single high-frequency excitation field $\mathbf{H}_{e}=\mathbf{H}_{z}$, records the fundamental magnetization response at $f_{z} \pm n f_{x, y}$, and excludes the higher harmonic components. Therefore, it can use a very low excitation-field amplitude, whereas narrowband MPI requires a large field amplitude for the $f_{0}$ and $f_{1}$ fields and a bandstop filter for the fundamental $\left(f_{0}\right)$ due to direct feedthrough contamination from the excitation fields. However, in the modulated MPI, $\mathbf{H}_{x} \perp \mathbf{H}_{y} \perp \mathbf{H}_{z}$ fields must be satisfied to effectively modulate the highfrequency magnetization response at the FFP with the low-frequency movement of the FFP itself. The receive coil is accordingly placed on the $z$ axis to capture $M_{z}^{*}(t)$.

In the case of $\mathbf{H}_{e}=\mathbf{H}_{0}+\mathbf{H}_{1}=\mathbf{H}_{x}$ with $\mathbf{H}_{0} \| \mathbf{H}_{1}$ (as found in narrowband MPI) [9], the resulting magnetization $\mathbf{M}$ will be associated mainly with the modulation of $\mathbf{M}_{x}$; thus, $\mathbf{M}_{y}$ attributed to the slow sample movement $\mathbf{y}$ or $\mathbf{H}_{y}$-steered FFP movement along the $y$ axis may be insignificant in comparison with M. Meanwhile, the perpendicularity of the excitation field to the FFP-trajectory plane results in $d V_{z}(t)=-\mu_{0} K\left[a_{x} M_{x}(t)+a_{y} M_{y}(t)\right]$ due to the time-varying tracer concentration $c(t)$ as during the FFP movement. $d V_{z}$ can be later plotted in an $(x, y)$ grid as a $2 \mathrm{D}$ composite image of the $f_{z} \pm n f_{x, y}$ harmonic product. From Fig. 6, our instrumentation setup of the modulated MPI includes mainly triggered function generators (i.e., AFG1062, Tektronix Inc., and G5100A, Picotest), high-speed amplifiers (i.e., HSA4011 and HSA4014, NF Corporation), a low-noise amplifier and a programmable filter (i.e., 5307 and 3627, NF Corporation), and an oscilloscope for data acquisition (i.e., MSO54, Tekronix Inc.). We use Litz coils to produce $\mathbf{H}_{x}$ and $\mathbf{H}_{y}$ with less frequency dependence of their impedance characteristics, thus requiring no resonant matching for the coils to be tuned below $5 \mathrm{kHz}$. However, we need to finely adjust the impedance of the induction and receive coils for $\mathbf{M}_{z}^{*}$ measurement expressed by $V_{z}$. We set $f_{z}=1 \mathrm{MHz}$ so that the Néel relaxation significantly contribute to $\mathbf{M}_{z}^{*}$ and increases the SNR of $V_{z}$.

\section{RESULTS AND DISCUSSION}

Unique relaxation behavior of magnetic nanoparticles is beneficial for realizing MPI. While the nonlinear field-dependent dynamic magnetization resulting 
(a)

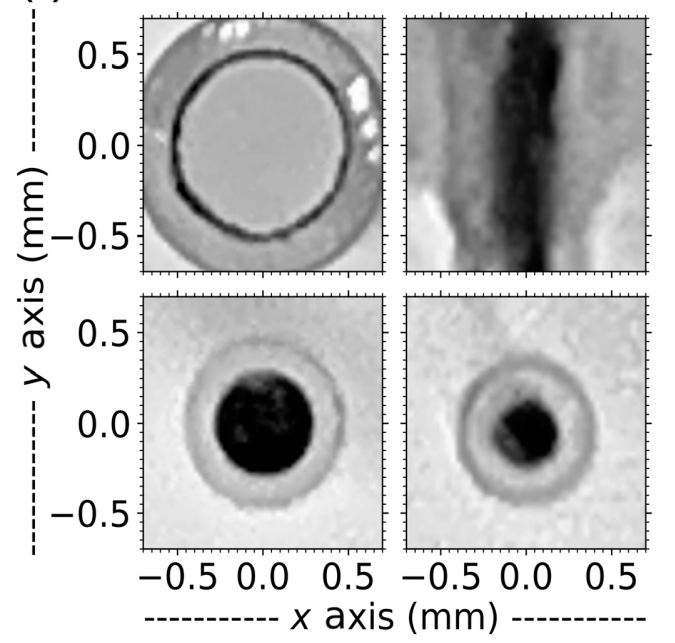

(b)

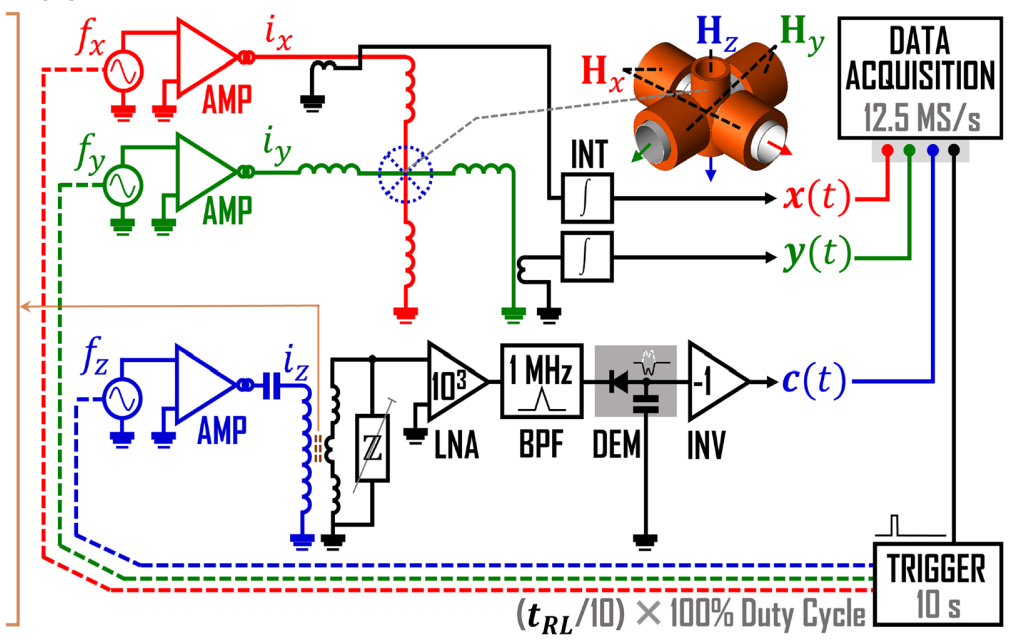

FIG. 6. (a) Solid-phantom photographs. We use three different geometries of the phantoms: 0.05-mm-thick ring with 1-mm diameter, 0.4-mm-wide rod, and a solid circle with diameters of 0.6 and $0.4 \mathrm{~mm}$. (b) Hardware setup. Three function generators are synchronously triggered at $10 \mathrm{~s}$ to produce ac waveforms at $f_{x}, f_{y}$, and $f_{z}$ fed to the transconductance amplifiers (AMP). The resulting $i_{x}, i_{y}$, and $i_{z}$ currents pass through the coils to generate $H_{x}, H_{y}$, and $H_{z}$ magnetic fields, where $i_{x}$ and $i_{y}$ are probed to estimate $x(t)$ and $y(t) . M_{z}^{*}(t)$ is measured as $V_{z}(t)$ by the gradiometric receive coil with careful impedance tuning. Before data acquisition, the signal is amplified at low noise, filtered at 1-MHz passband, and demodulated to estimate $c(t)$. Here, LNA, BPF, DEM, INV, and INT represent low noise amplifier, band-pass filter, signal demodulator, inverter, and integrator, respectively. We sample the demodulated signal at 12.5 MS/s while performing envelope tracking during the acquisition.

in the harmonic responses is the fundamental property exploited by regular MPI, frequency-dependent magnetization enables the modulated MPI to use the linear response in the low-field regime. We previously showed that the fast moment relaxation can carry the magnetization response induced by the moving FFP; thus, the magnetization sampling rate corresponds to the Néel relaxation frequency. Since moment dynamics may exist at a high dc bias field [58], the modulated MPI is effective under high field gradient (e.g., above $20 \mathrm{~T} / \mathrm{m}$ ). Nevertheless, when a low static field gradient (e.g., below $2 \mathrm{~T} / \mathrm{m}$ ) is used, the modulated MPI may be practically inferior to standard MPI. It relies on Néel relaxation, which leads to less static-field-dependent magnetization as compared with the magnetization harmonics. Furthermore, Fig. 2(a) reveals that the high-frequency magnetic susceptibility is significantly smaller than the magnetic susceptibility in low-frequency regime. Therefore, considering that the susceptibility ratio affects the amplitude sensitivity of the recorded signal, $f_{x}$ and $N_{D}$ become important parameters for refining the MPI resolution under low field gradient.

\section{A. FFP-trajectory density}

The FFP-trajectory density $N_{D}$ generally describes the scan coverage of the sample. Large $N_{D}$ minimizes the spatial sampling vacancies, where the corresponding signals are often interpolated to accommodate a MPI system matrix [59]. For the Lissajous FFP trajectory, the pattern can be created by $\mathbf{H}_{x} \perp \mathbf{H}_{y}$ with $f_{x}<f_{y}$; thus, a small frequency difference $\Delta f_{x y}=f_{y}-f_{x}$ results in large $N_{D}$. Here, adjusting $f_{x}$ and $N_{D}$ is important to optimize $t_{R L}=N_{D} / 2 f_{x}$, which represents the signal acquisition time for a single (nonrepetitive) frame of the FOV. For constant $N_{D}=200$, $f_{x}=0.5 \mathrm{kHz}$ gives greater spatial resolution than $f_{x}=$ $4 \mathrm{kHz}$, where the respective $t_{R L}$ is $200 \mathrm{~ms}, 8$-fold larger than that at $f_{x}=4 \mathrm{kHz}$. Long $t_{R L}$ leads to a large $1-\mathrm{MHz}-$ sampled magnetization response, which is effective for further data averaging. Moreover, low-frequency $H_{x}$ and $H_{y}$ induces a larger magnetic response than high-frequency fields. Even though signal amplification of the electromotive force is proportional to the applied frequency, Eq. (10) takes the resulting $M_{x}(t)$ and $M_{y}(t)$ into account and notes that $\dot{M}_{x}(t)$ and $\dot{M}_{y}(t)$ are irrelevant in the case of highfrequency $H_{z}$. However, our concern is to minimize $t_{R L}$ but provide a comparable spatial resolution such in the case of $f_{x}=2 \mathrm{kHz}$. Therefore, it is necessary to further investigate the effect of changing $N_{D}$.

From Fig. 7, reducing $N_{D}$ from 200 to 50 results in very low spatial resolution although $t_{R L}$ is decreased to 12.5 ms. Similarly, $N_{D}>300$ shows no significant increase of the spatial resolution as compared with $N_{D}=200$. We previously defined that the maximum trajectory density $N_{D, \max }$ equals $f_{z} / f_{x}$ [33], where $N_{D, \max }=400$ for $f_{x}=2$ $\mathrm{kHz}$ and $f_{z}=1 \mathrm{MHz}$. Figure 7 implies that $N_{D}$ should depend on how large the FOV is to further optimize the resolution. Large $N_{D}$ increases $t_{R L}$, which may negatively affect the signal processing and result in large spatial noise 


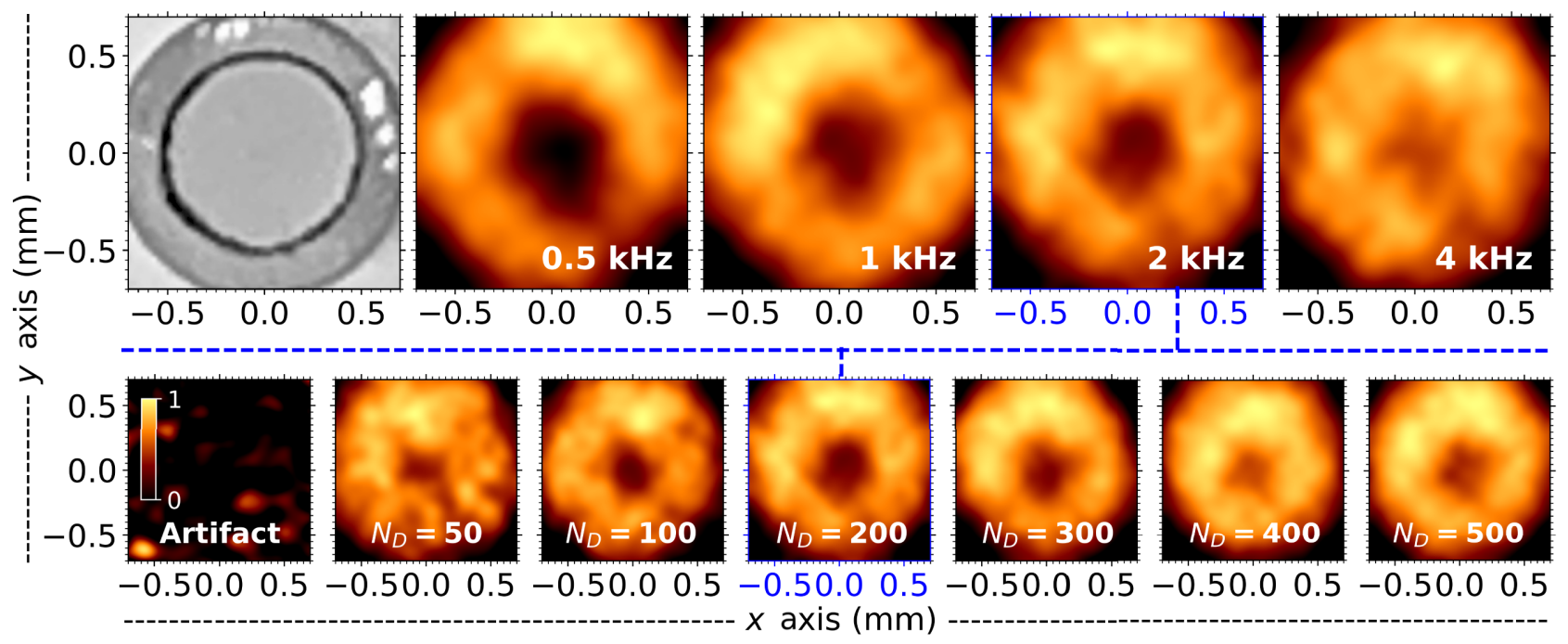

FIG. 7. Reconstructed images of $1 \mathrm{~mm}$ solid Resovist ring phantom for different $f_{x}$ and $N_{D}$. On increasing $f_{x}$ up to $4 \mathrm{kHz}$ at constant $N_{D}=200$, the spatial resolution becomes low. Similarly, decreasing $N_{D}$ from 200 to 50 at constant $f_{x}=2 \mathrm{kHz}$ appears to reduce the resolution even though further increase of $N_{D}$ above 300 does not increase it. A photograph of the phantom is shown in the top-left panel, whereas the artifact from the phantom-free scan at $N_{D}=200$ is shown in the bottom-left panel.

(i.e., artifact corresponding to the sample-free signal). Here $N_{D}=200$ appears sufficient to image the $1.4 \times 1.4 \mathrm{~mm}^{2}$ FOV with approximately-0.9-mm spatial resolution under $2 \mathrm{~T} / \mathrm{m}$ field gradient (discussed further in Appendix B). In the modulated MPI, the spatial resolution is proportional to the pixel size $d_{x}=d_{y}$, which is defined as the length of the maximum FFP displacement over the trajectory density, $d_{x}=2 H_{x, 0} /\left(N_{D} G_{x}\right)$ and $d_{y}=2 H_{y, 0} /\left(N_{D} G_{y}\right)$ [33]. In the case of a large field gradient, the spatial resolution may be close to the pixel size. However, it is difficult to give an exact value for the spatial resolution since the MPI instrumentation system contributes to the SNR; thus, an empirical estimate is more realistic.

Previous analysis of the effect of $N_{D}$ on image quality from an $x$-space MPI perspective shows that the SNR converges as $N_{D}$ increases [60]. In the case of our modulated MPI scanner, $f_{z}$ is used as the frequency to synchronize the MPI signal [i.e., $\left.d V_{z}(t)\right]$ with the FFP trajectory. Thus, the maximum pixel number for a 1D FFP scan is given by $N_{D, \max }=T_{x} / T_{z}=f_{z} / f_{x}$, where $T_{x}$ and $T_{z}$ are periods of the scan waveform and the signal initially sampled at $12.5 \mathrm{MS} / \mathrm{s}$, respectively. Here $N_{D \text {, } \max }$ represents the optimum resolution, and further increasing $N_{D}>N_{D \text {,max }}$ may not practically increase the resolution, but may possibly result in more noise in the acquired signal. Figure 8 convinces us that the SNR is linear with $f_{x}$ but appears to be saturated above $N_{D, \max }$. However, we do not take the spatial noise into account when expressing the SNR as $20 \log _{10}\left(d V_{z, s} / d V_{z, r}\right)$. Here $d V_{z, s}$ and $d V_{z, r}$ are the maximum pixel intensity and the $1.7-\mu \mathrm{V}$ noise level, respectively. Therefore, the SNR needs to be correlated with the $t_{R L}$ dependence of $d V_{z, s}$. Figure 8 further highlights low
SNR efficiency either at low $f_{x}$ or at high $N_{D}$ in terms of $d V_{z, s} / \sqrt{t_{R L}}$ relative to $d V_{z, r} / \sqrt{t_{R L}}$.

\section{B. Static field gradient}

On the basis of the derivative of the Langevin function at the FWHM, standard MPI predicts an approximate value of the intrinsic 1D MPI resolution on the $x$ axis as $d_{x}^{R}=$ $4 k_{B} T /\left(m G_{x}\right)$, emphasizing the mean magnetic moment of the particles and the field gradient [12]. In the case of
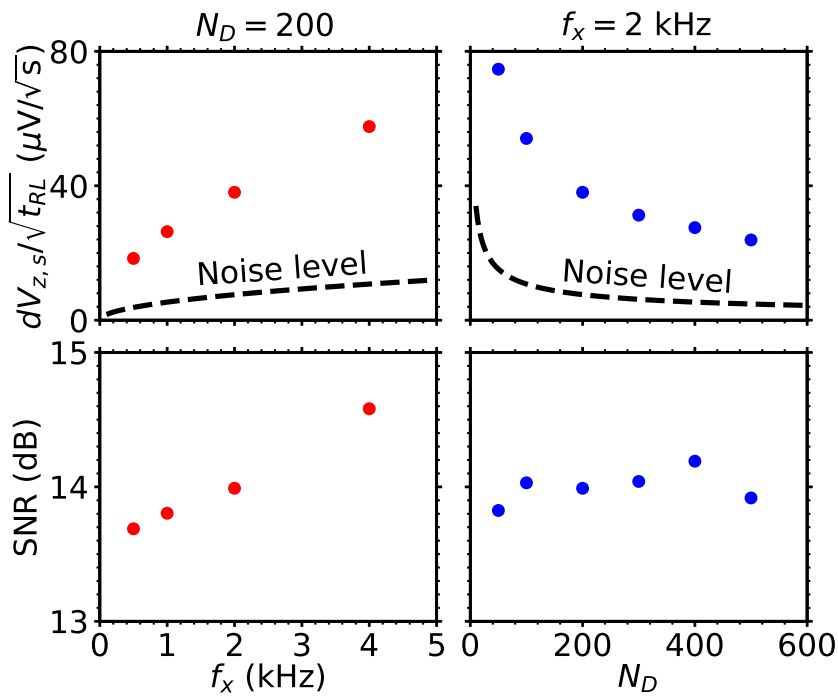

FIG. 8. Maximum pixel intensity $d V_{z, s}$ relative to the record length and 1-MHz noise level for different $f_{x}$ and $N_{D}$. The calculated SNR reflects the image quality at constant maximum noise level $d V_{z, r}=1.7 \mu \mathrm{V}$. 
Resovist containing mainly 7-nm maghemite nanoparticles, a $2 \mathrm{~T} / \mathrm{m}$ field gradient should give $d_{x}^{R}=15.6 \mathrm{~mm}$ for a weighted mean $m_{p}$ of $5.31 \times 10^{-19} \mathrm{~J} / \mathrm{T}$ for the solid sample and $d_{x}^{R}=1.3 \mathrm{~mm}$ for a weighted mean $m_{c}$ of $6.26 \times 10^{-18} \mathrm{~J} / \mathrm{T}$ for the liquid sample; $m_{p}$ and $m_{c}$ are calculated from the inset in Fig. 1(a). In comparison, Fig. 4 highlights the increased resolution for the solid sample by optimization of the scan parameters of the modulated MPI under $2 \mathrm{~T} / \mathrm{m}$. It may be necessary to further decrease the field gradient to be compatible with submillimeter imaging. Even though a low field gradient theoretically reduces the spatial resolution [61], it can inhibit magnetic separation when the tracers are moved by a large magnetic force.

The field gradient is used to effectively saturate the magnetic response of the tracers outside the FFP as defined by

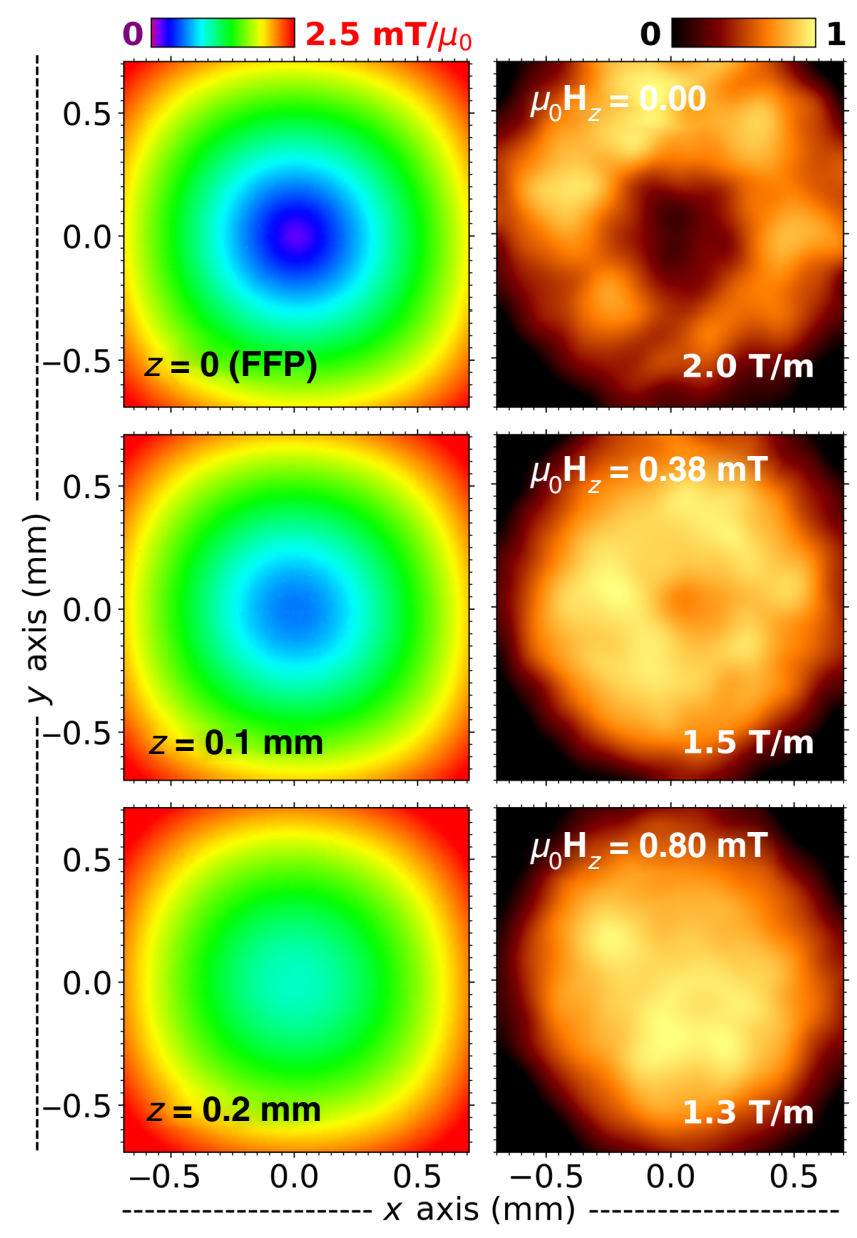

FIG. 9. Effect of field gradient on the reconstructed image. The left panels depict the spatial distribution of the static field relative to the $z$ position simulated by JMAG-Designer 19.0, whereas the right panels display the corresponding image of the $0.1-\mathrm{mm}$ solid Resovist ring phantom with a height of approximately $0.2 \mathrm{~mm}$. $z=0$ corresponds to the FFP where the middle slice of the phantom is imaged. Increasing $z=0.2 \mathrm{~mm}$ above the FFP reduces the field gradient to $1.3 \mathrm{~T} / \mathrm{m}$. the PSF; thus, the spatial resolution strongly depends on the field gradient [5]. Figure 9 confirms that a low field gradient results in low spatial resolution. Here the ring phantom is translated along the $z$ axis, while the FFPtrajectory plane remains at $z=0$. The phantom height is $0.2 \mathrm{~mm}$, and $z=0, z=0.1$, and $z=0.2 \mathrm{~mm}$ represent the middle slice, the top slice, $0.1 \mathrm{~mm}$ from from top slice of the phantom, respectively. At $z=0$, where the FFP exists under $G_{x}=G_{y}=2 \mathrm{~T} / \mathrm{m}$, the geometry of ring phantom can be well replicated. However, at $z=0.1 \mathrm{~mm}$ above the FFP, a slight decrease in the field gradient $\left(G_{x}=\right.$ $\left.G_{y}=1.5 \mathrm{~T} / \mathrm{m}\right)$ appears to reduce the resolution; similarly at $z=0.2 \mathrm{~mm}\left(G_{x}=G_{y}=1.3 \mathrm{~T} / \mathrm{m}\right)$. The presence of the $H_{z}$ bias field may also contribute to reducing the spatial resolution. From Fig. 9, the simulation result shows that the tracers may experience $H_{z}=0.38 \mathrm{mT} / \mu_{0}$ at $0.1 \mathrm{~mm}$ above the FFP, in addition to the reduced field gradient. Since all tracers are already statically magnetized, the modulated field gradient is no longer attributable to the FFP, but is a point of minimum field. As a consequence, the modulated MPI cannot maintain the phantom geometry when a low field gradient is applied with the bias field.

\section{Phantom geometry}

Visualizing the phantom geometry on an accurate scale is the most-important task of an imaging system. Similarly, MPI requires high spatial resolution to reconstruct the phantom image with morphology identical to that of the original object. The modulated MPI offers submillimeter resolution at a low field gradient, and thus should be capable of tracking a finite displacement of the samples. Figure 10(a) further demonstrates that the modulated MPI can track both a $0.1 \mathrm{~mm}$ shift of the solid ring phantom on the $x$ axis and a $10^{\circ}$ rotation of the solid bar phantom. Although each geometry of the solid phantom is qualitatively preserved, Fig. 10(b) indicates image distortion in the case of the liquid phantoms, particularly for large size. The distortion can be further observed when one images two-adjacent $0.6-\mathrm{mm}$ liquid phantoms with a center-to-center distance of $0.9 \mathrm{~mm}$; nevertheless, the modulated MPI can obviously identify two neighboring 0.4-mm liquid phantoms for the same distance.

In the case of a solid phantom, both low-frequency and high-frequency fields induce a magnetization response that originates from the Néel relaxation, and thus either $M_{x}(t)$ or $M_{y}(t)$ is effectively coupled with $M_{z}(t)$ without a phase delay relative to the low-frequency excitation fields. The linear magnetization response further leads to more-broadened images than those observed in the liquid phantoms [Fig. 10(b)]. Since the Brownian and Néel relaxations coexist in the liquid phantom [62], they result in different phase delays of low-frequency and high-frequency magnetization responses relative to the applied fields. The resulting phase delay between $M_{z}^{*}(t)$ and $H_{x}(t)$ [as well 
(a)

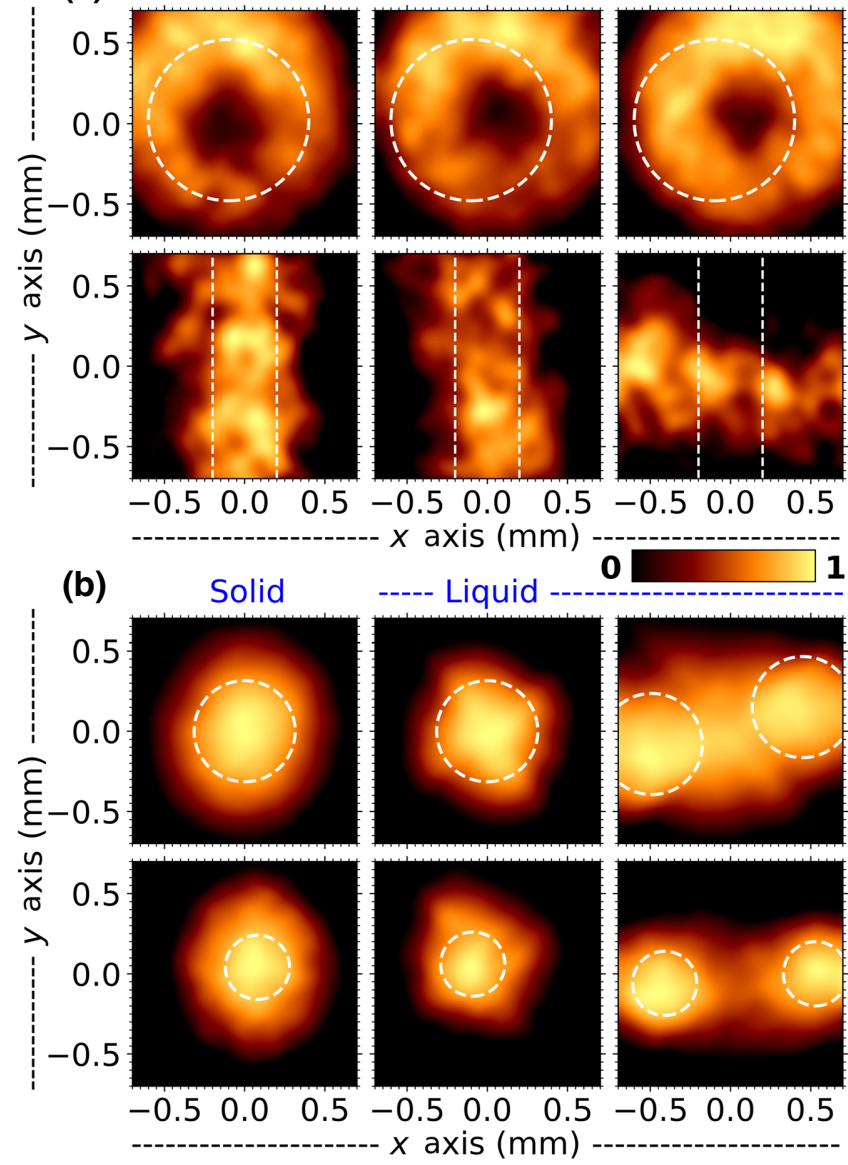

FIG. 10. (a) Reconstructed images of solid Resovist samples with different positions and orientations. The solid ring phantom mechanically shifted on the $x$ axis in $0.1-\mathrm{mm}$ intervals can be well imaged by the modulated MPI, and similarly for the rotated solid rod phantom with $0.4-\mathrm{mm}$ width (second row). (b) Reconstructed circular-phantom images of solid and liquid Resovist samples with different diameters: $0.6 \mathrm{~mm}$ (first row) and 0.4 $\mathrm{mm}$ (second row). The images of solid phantoms appear to have geometry similar to that of the real objects, whereas the distorted images of the liquid phantoms are attributed to the Brownian relaxation effects. The dashed line represents the boundary of the phantom geometry.

as $H_{y}(t)$ ] contributes to the image distortion [Fig. 10(b)] even though the Brownian particle rotations increase the magnetic susceptibility and further increase the resolution. Therefore, image correction by minimizing the magnetization delay is necessary in the modulated MPI to improve the image reconstruction.

\section{Phase shifting}

Figure 10(b) shows that the spatial resolution achieved is too low to clearly distinguish two neighboring $0.6-\mathrm{mm}$ circular phantoms of the liquid samples. Since we use a (a)
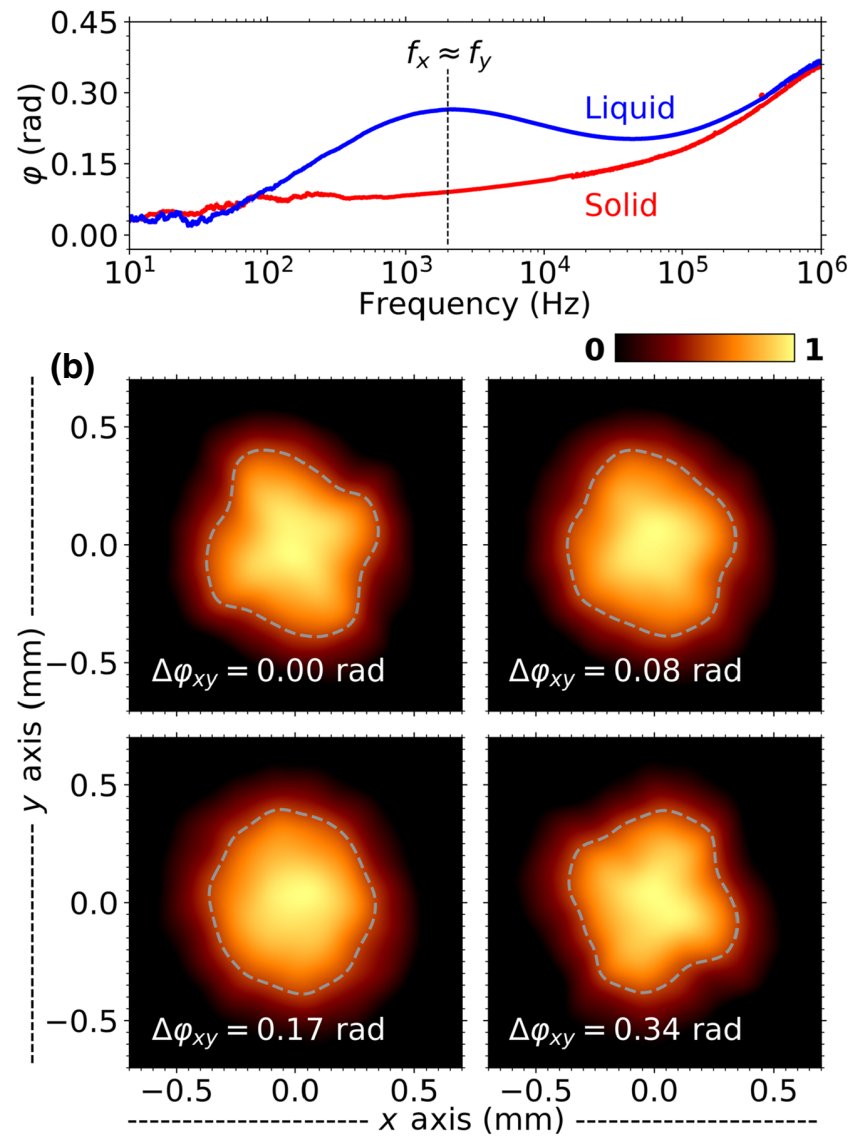

FIG. 11. (a) Phase-delay $(\varphi)$ characteristics of Resovist relative to the applied frequency and (b) corrected images of the 0.6-mm circular liquid phantom based on phase shifting. In the liquid sample, the Brownian relaxation dominantly contributes to greater phase delay at low frequency than in the solid sample. The resulting image distortion can be fixed by the phase shifting of $d V_{z}(t)$ at $\Delta \varphi_{x y}=0.17 \mathrm{rad}$, which corresponds to the phase difference between magnetization responses of the liquid and solid samples at excitation frequencies $f_{x} \approx f_{y}$.

field gradient more than 10 times lower than in our previous setup [33], we cannot expect high resolution below $0.1 \mathrm{~mm}$. In this case, the small $a_{x} M_{x}(t)$ and $a_{y} M_{y}(t)$ values determining the amplitude modulation of $\dot{M}_{z}(t)$ may be responsible for the blurry image attributed to low $d V_{z}(t)$. Furthermore, Fig. 11(a) indicates that the Brownian relaxation in the liquid samples potentially distorts the PSF due to a large phase delay at low excitation frequencies. At $f_{x} \approx f_{y}$, the phase difference between the liquid and solid samples, $\Delta \varphi_{x y}$, is $0.17 \mathrm{rad}$. To correct the distorted images of the liquid samples, it is necessary to shift $d V_{z}(t)$ by $\Delta t=\left(\Delta \varphi_{x y} T_{x y}\right) / 2 \pi$, where $T_{x y} \approx 1 / f_{x} \approx 1 / f_{y}$ is the period of the FFP-steering fields.

Empirically, Eq. (4) generalizes the magnetization response of a semirigid-dipole system rotatable in a liquid medium as $M(t)=M^{N}(t)+M^{B}(t)$ [43]. For the given 
$H_{x}(t)=H_{x, 0} \cos \omega_{x} t$, the Néel relaxation of core particles contributes to $M_{x}(t)$ in terms of $M_{x}^{N}(t)=M_{x, 0}^{N} \cos \left(\omega_{x} t-\right.$ $\left.\varphi_{x}^{N}\right)$. Similarly, the Brownian relaxation of hydrodynamic nanoclusters leads to $M_{x}^{B}(t)=M_{x, 0}^{B} \cos \left(\omega_{x} t-\varphi_{x}^{B}\right)$. Here $\varphi_{x}^{N}$ and $\varphi_{x}^{B}$ are the field-relative phase delay attributed to the Néel and Brownian relaxations at $f_{x}$, respectively. Meanwhile, high-frequency $H_{z}(t)=H_{z, 0} \cos \omega_{z} t$ induces $M_{z}(t)=M_{z, 0}^{N} \cos \left(\omega_{z} t-\varphi_{z}^{N}\right)$, which is further modulated by $M_{x}(t)$. The Néel relaxation leads to $M_{z}(t)$ delayed by $\varphi_{z}^{N}$ relative to $H_{z}(t)$. In the case of the liquid sample where $M_{x, 0}^{B}>M_{x, 0}^{N}$, the resulting $M_{z}^{*}(t)$ has a large phase delay relative to $H_{x}(t)$ [as well as $x(t)$ ], which corresponds to $\varphi_{x}^{B}-\varphi_{x}^{N}$. Therefore, under the orthogonal $x-y$ excitation fields with $f_{x} \approx f_{y}$, the field-relative phase delay defined as $\Delta \varphi_{x y}=\varphi_{x}^{B}-\varphi_{x}^{N}$ is necessarily applied to compensate the Brownian relaxation effects.

From Fig. 11(b), it appears that the reconstructed image of the $0.6-\mathrm{mm}$ circular liquid phantom is distorted without phase correction $\left(\Delta \varphi_{x y}=0.00 \mathrm{rad}\right)$. The circular geometry is then obtained by our phase shifting $\left[d V_{z}(t+\Delta t)\right]$ at $\Delta \varphi_{x y}=0.17 \mathrm{rad}$, which is the phase difference between the magnetization responses of the liquid and solid samples at $2 \mathrm{kHz}$. Increasing $\Delta \varphi_{x y}$ to $0.34 \mathrm{rad}$ results in further image distortion. A similar time shifting of the signal was previously proposed for $x$-space MPI reconstruction to compensate the delayed FFP velocity by half the relaxation time [63]. However, for $\tau_{B}=2.33 \times 10^{-4}$ $\mathrm{s}$ of Resovist, the velocity compensation $\tau_{B} / 2$ larger than $\Delta t=1.35 \times 10^{-5}$ for $\Delta \varphi_{x y}=0.17$ seems improper to correct the distorted image in the case of modulated MPI. Unlike the phase weighting, which typically multiplies the original signal by the phase delay (e.g., $d V_{z} \cos \varphi_{x y}$ and $d V_{z} \sin \varphi_{x y}$ ) [64], the phase shifting affects neither the signal amplitude nor the spatial resolution. This strategy highlights that the modulated MPI can solve the relaxation effects while offering submillimeter spatial resolution under low field gradient.

\section{CONCLUSIONS}

We introduce an alternative MPI strategy that exploits the frequency-dependent relaxation characteristics of superparamagnetic nanoparticles, instead of nonlinear field-dependent dynamic magnetization. Like the standard system, the modulated MPI is capable of producing 1D, $2 \mathrm{D}$, and 3D images of magnetic tracers with a simplified setup. To achieve submillimeter resolution under low field gradient, it is necessary to use distinguishable excitation fields which two-dimensionally steer the FPP trajectory at low frequencies and capture the modulated Néel relaxation at high frequency. By optimizing the excitation frequencies, trajectory density, and field gradient, our time-domain image-reconstruction method promises a spatial resolution of at least $0.9 \mathrm{~mm}$ at a field gradient of $2 \mathrm{~T} / \mathrm{m}$ to visualize solid phantoms with geometrically preserved images, where standard MPI may face the disadvantage of processing more linear magnetic responses of the immobilized tracers. We demonstrate that 2D images of a 1-mm solid Resovist ring phantom with $0.05-\mathrm{mm}$ thickness, as well as a $0.4-\mathrm{mm}$ solid bar phantom, can be well imaged on a $1.4 \times 1.4 \mathrm{~mm}^{2}$ FOV to track their finite mechanical displacements. Furthermore, the modulated MPI introduces phase shifting to resolve the image distortion due to Brownian relaxation effects of the liquid phantom, which can be useful to handle large magnetic nanotracers. These promising features may promote the modulated MPI system as a complementary instrumentation system for submillimeter imaging of cells or small-animal models.

\section{ACKNOWLEDGMENTS}

This work was partially supported by JSPS KAKENHI Grants No. 15H05764, No. 17H03275, and No. 20H05652, as well as the Standard Program of JSPS Postdoctoral Fellowship.

\section{APPENDIX A: SYMMETRICAL FIELD GRADIENT}

A field gradient is used to provide the selection field and create the FFP, thus enabling the spatial encoding of the magnetic signal. As shown in Figs. 3 and 12, we are
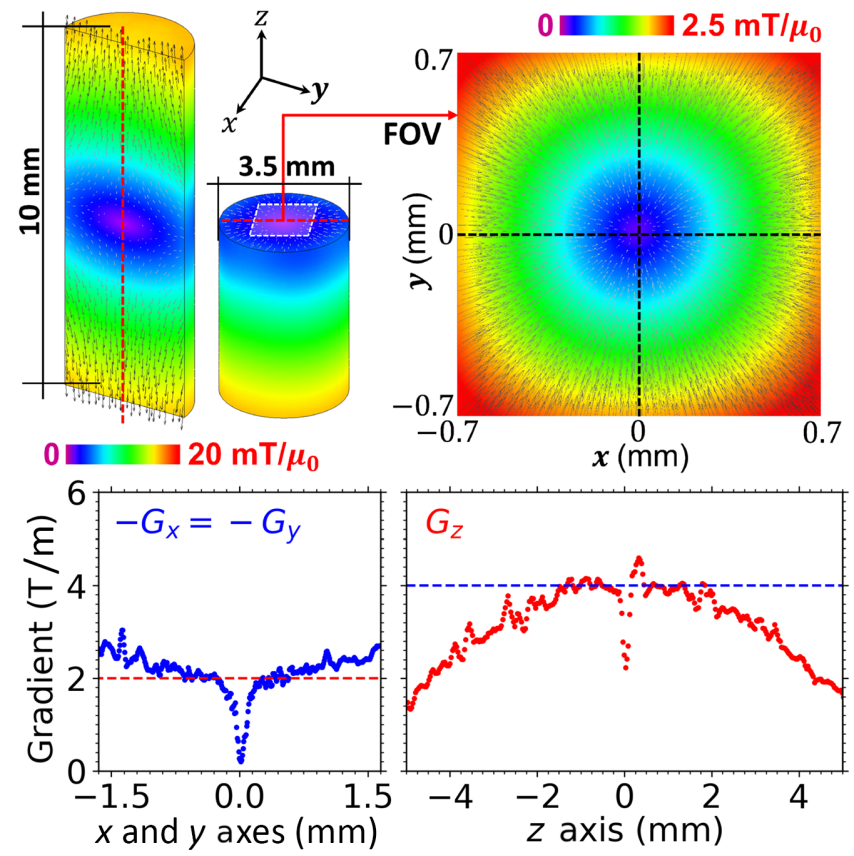

FIG. 12. Magnetic field distribution and gradient around the FFP simulated by JMAG-Designer 19.0. Two sets of permanent magnets installed on the $x$ and $y$ axes create a symmetrical field gradient that locates the FFP at the center of the magnet configuration. The software appears to underestimate the gradient near the FFP due to the limit of the mesh size. At $-1.3 \mathrm{~mm} \leq$ $x, y, z \leq 1.3 \mathrm{~mm}, G_{x}=G_{y}=-0.5 G_{z}$ equals $2 \mathrm{~T} / \mathrm{m}$ if the vector of the fields is taken into account. 
able to create a symmetrical field gradient in the $x-y$ plane (i.e., $G_{x}=G_{y}$ ) by installing four permanent magnets with all north poles facing each other and pointing to the FFP. In this magnet configuration, the resulting gradients differ in their distance relative to the FFP, which further satisfies Maxwell's equation in terms of $G_{x}=G_{y}=-0.5 G_{z}=$ $2 \mathrm{~T} / \mathrm{m}$ at $-1.3 \mathrm{~mm} \leq x, y, z \leq 1.3 \mathrm{~mm}$ across the FFP [65]. These FFP-displacement constraints work as a maximum expandable boundary of the FOV in our MPI scanner; however, we practically use $-0.7 \mathrm{~mm} \leq x, y \leq 0.7 \mathrm{~mm}$ while centering the FFP at $z=0$ with very low $H_{z, 0}$.

\section{APPENDIX B: SPATIAL RESOLUTION}

From the 1D FFP scan, Fig. 3 has already identified that the spatial resolution of our modulated MPI scanner should be less than $1.5 \mathrm{~mm}$, which corresponds to the center-tocenter distance of two neighboring circular liquid phantoms. To estimate the spatial resolution more accurately, we analyze the reconstructed images of ring phantoms by comparing them with the original photograph. As shown in Fig. 13, the reconstructed 1-mm-ring-phantom image blurs, and it becomes thicker than the actual object. The inner blurring boundary is $0.5 \mathrm{~mm}$ in diameter, and thus we roughly estimate the spatial resolution as the actual ring diameter minus the diameter of the blurring boundary,

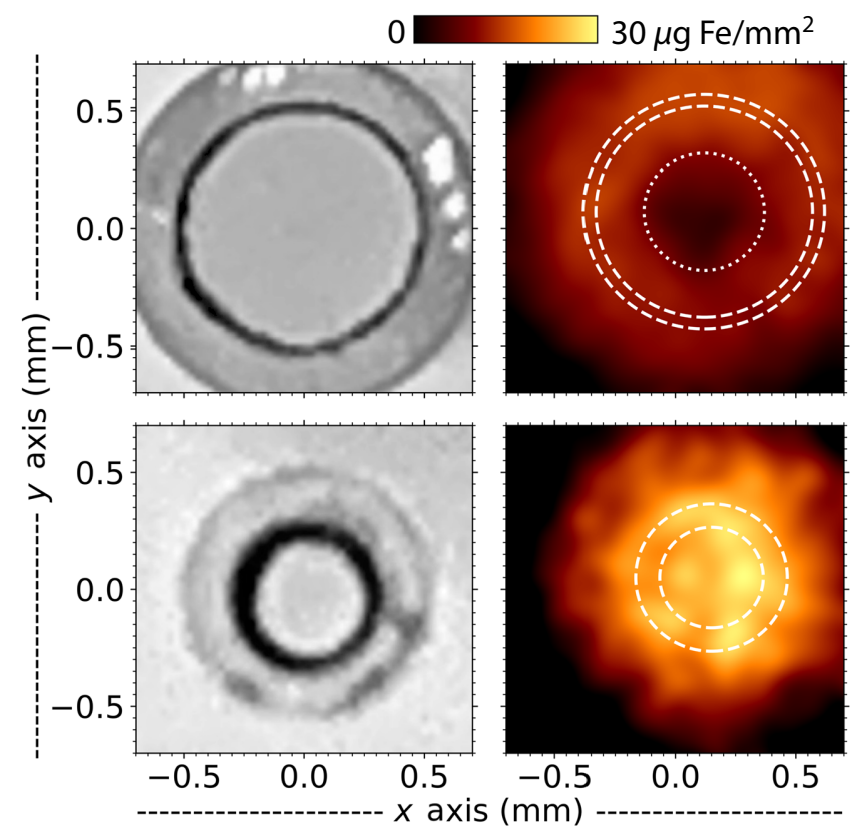

FIG. 13. Reconstructed images of the ring phantoms with different sizes and thicknesses: 1 and $0.05 \mathrm{~mm}$ and 0.6 and $0.1 \mathrm{~mm}$. The spatial MPI resolution is then qualitatively evaluated from the inner diameter of the 1-mm-ring-phantom image (dotted line) displaying the blurring boundary from the actual photograph (dashed line). The 0.6-mm-ring-phantom image further confirms the resolution must not be greater than $0.5 \mathrm{~mm}$. which is approximately $0.5 \mathrm{~mm}$. This value represents a minimum center-to-center distance that causes two phantom images to nearly overlap with each other. We later image a $0.6-\mathrm{mm}$ ring phantom with $0.1-\mathrm{mm}$ thickness, so its center-to-center distance is $0.5 \mathrm{~mm}$. Figure 13 (second row) shows that the reconstructed image has no hole at its center as found in the actual geometry. Figure 13 implies that the spatial resolution must not be greater than $0.5 \mathrm{~mm}$.

Empirically, we estimate that the spatial resolution of our modulated MPI scanner is $0.5 \mathrm{~mm}<d_{x}, d_{y}<1.5 \mathrm{~mm}$. For a more-accurate value, we refer to Fig. 10(b), which shows a good separation of two-neighboring 0.4-mm circular images with a center-to-center distance of $0.9 \mathrm{~mm}$; thus, $d_{x}=d_{y}=0.9 \mathrm{~mm}$. Nevertheless, the spatial resolution appears to depend on the tracer concentration. For a large amount of tracers, the signal achieved will be sufficiently high such that the reconstructed images of two adjacent phantoms may overlap [Fig. 10(b), first row], whereas a low tracer concentration may blur the images due to a low SNR. The tracer concentration can be estimated by dividing the sample mass by the area of the actual geometry as shown in Fig. 13. Meanwhile, low spatial resolution may also correlate with broad (primary and secondary) size distributions of the tracers. In the case of a narrow size distribution, the relaxation spectra of the tracers satisfy a single relaxation time of the Debye model with symmetrical $\chi^{\prime \prime}\left(\chi^{\prime}\right)$ behavior [58]. Therefore, we expect a large susceptibility difference $\Delta \chi$ between $\chi(\omega \tau \ll 1)$ and $\chi(\omega \tau \gg 1)$, which can increase the amplitude sensitivity of the modulated MPI signal to obtain high image contrast for monodisperse tracers.

[1] B. Gleich and J. Weizenecker, Tomographic imaging using the nonlinear response of magnetic particles, Nature 435, 1214 (2005).

[2] E. Y. Yu, P. Chandrasekharan, R. Berzon, Z. W. Tay, X. Y. Zhou, A. P. Khandhar, R. M. Ferguson, S. J. Kemp, B. Zheng, P. W. Goodwill, M. F. Wendland, K. M. Krishnan, S. Behr, J. Carter, and S. M. Conolly, Magnetic particle imaging for highly sensitive, quantitative, and safe in vivo gut bleed detection in a murine model, ACS Nano 11, 12067 (2017).

[3] C. P. Bean and J. D. Livingston, Superparamagnetism, J. Appl. Phys. 30, S120 (1959).

[4] J. B. Weaver, A. M. Rauwerdink, C. R. Sullivan, and I. Baker, Frequency distribution of the nanoparticle magnetization in the presence of a static as well as a harmonic magnetic field, Med. Phys. 35, 1988 (2008).

[5] T. Knopp and T. M. Buzug, Magnetic Particle Imaging: An Introduction to Imaging Principles and Scanner Instrumentation (Springer-Verlag, Berlin, Heidelberg, 2012), 1 st ed.

[6] J. Weizenecker, B. Gleich, J. Rahmer, H. Dahnke, and J. Borgert, Three-dimensional real-time in vivo magnetic particle imaging, Phys. Med. Biol. 54, L1 (2009). 
[7] P. Vogel, M. A. Rückert, P. Klauer, W. H. Kullmann, P. M. Jakob, and V. C. Behr, First in vivo traveling wave magnetic particle imaging of a beating mouse heart, Phys. Med. Biol. 61, 6620 (2016).

[8] P. Ludewig, N. Gdaniec, J. Sedlacik, N. D. Forkert, P. Szwargulski, M. Graeser, G. Adam, M. G. Kaul, K. M. Krishnan, R. M. Ferguson, A. P. Khandhar, P. Walczak, J. Fiehler, G. Thomalla, C. Gerloff, T. Knopp, and T. Magnus, Magnetic particle imaging for real-time perfusion imaging in acute stroke, ACS Nano 11, 10480 (2017).

[9] P. W. Goodwill, G. C. Scott, P. P. Stang, and S. M. Conolly, Narrowband magnetic particle imaging, IEEE Trans. Med. Imaging 28, 1231 (2009).

[10] J. Rahmer, A. Halkola, B. Gleich, I. Schmale, and J. Borgert, First experimental evidence of the feasibility of multicolor magnetic particle imaging, Phys. Med. Biol. 60, 1775 (2015).

[11] Y. Muslu, M. Utkur, O. B. Demirel, and E. U. Saritas, Calibration-free relaxation-based multi-color magnetic particle imaging, IEEE Trans. Med. Imaging 37, 1920 (2018).

[12] P. W. Goodwill and S. M. Conolly, The x-space formulation of the magnetic particle imaging process: 1-D signal, resolution, bandwidth, SNR, SAR, and magnetostimulation, IEEE Trans. Med. Imaging 29, 1851 (2010).

[13] E. U. Saritas, P. W. Goodwill, G. Z. Zhang, and S. M. Conolly, Magnetostimulation limits in magnetic particle imaging, IEEE Trans. Med. Imaging 32, 1600 (2013).

[14] I. Schmale, J. Rahmer, B. Gleich, J. Kanzenbach, J. D. Schmidt, C. Bontus, O. Woywode, and J. Borgert, in Medical Imaging 2011: Biomedical Applications in Molecular, Structural, and Functional Imaging, edited by J. B. Weaver and R. C. Molthen (International Society for Optics and Photonics, SPIE, Bellingham, Washington, 2011), Vol. 7965, p. 263-268.

[15] C. Kaethner, M. Ahlborg, G. Bringout, M. Weber, and T. M. Buzug, Axially elongated field-free point data acquisition in magnetic particle imaging, IEEE Trans. Med. Imaging 34, 381 (2015).

[16] P. Vogel, M. A. Rückert, P. Klauer, W. H. Kullmann, P. M. Jakob, and V. C. Behr, Traveling wave magnetic particle imaging, IEEE Trans. Med. Imaging 33, 400 (2014).

[17] T. Knopp, S. Biederer, T. Sattel, J. Weizenecker, B. Gleich, J. Borgert, and T. M. Buzug, Trajectory analysis for magnetic particle imaging, Phys. Med. Biol. 54, 385 (2008).

[18] C. B. Top, A. Güngör, S. Ilbey, and H. E. Güven, Trajectory analysis for field free line magnetic particle imaging, Med. Phys. 46, 1592 (2019).

[19] M. H. Pablico-Lansigan, S. F. Situ, and A. C. S. Samia, Magnetic particle imaging: Advancements and perspectives for real-time in vivo monitoring and image-guided therapy, Nanoscale 5, 4040 (2013).

[20] E. Y. Yu, M. Bishop, B. Zheng, R. M. Ferguson, A. P. Khandhar, S. J. Kemp, K. M. Krishnan, P. W. Goodwill, and S. M. Conolly, Magnetic particle imaging: A novel in vivo imaging platform for cancer detection, Nano Lett. 17, 1648 (2017).

[21] M. Graeser, F. Thieben, P. Szwargulski, F. Werner, N. Gdaniec, M. Boberg, F. Griese, M. Möddel, P. Ludewig, D. van de Ven, O. M. Weber, O. Woywode, B. Gleich, and
T. Knopp, Human-sized magnetic particle imaging for brain applications, Nat. Commun. 10, 1936 (2019).

[22] Z. W. Tay, P. Chandrasekharan, A. Chiu-Lam, D. W. Hensley, R. Dhavalikar, X. Y. Zhou, E. Y. Yu, P. W. Goodwill, B. Zheng, C. Rinaldi, and S. M. Conolly, Magnetic particle imaging-guided heating in vivo using gradient fields for arbitrary localization of magnetic hyperthermia therapy, ACS Nano 12, 3699 (2018).

[23] P. Chandrasekharan, Z. W. Tay, D. Hensley, X. Y. Zhou, B. K. Fung, C. Colson, Y. Lu, B. D. Fellows, Q. Huynh, C. Saayujya, E. Yu, R. Orendorff, B. Zheng, P. Goodwill, C. Rinaldi, and S. Conolly, Using magnetic particle imaging systems to localize and guide magnetic hyperthermia treatment: Tracers, hardware, and future medical applications, Theranostics 10, 2965 (2020).

[24] B. Zheng, T. Vazin, P. W. Goodwill, A. Conway, A. Verma, E. U. Saritas, D. Schaffer, and S. M. Conolly, Magnetic particle imaging tracks the long-term fate of in vivo neural cell implants with high image contrast, Sci. Rep. 5, 14055 (2015).

[25] J. Rahmer, A. Antonelli, C. Sfara, B. Tiemann, B. Gleich, M. Magnani, J. Weizeneckerand, and J. Borgert, Nanoparticle encapsulation in red blood cells enables blood-pool magnetic particle imaging hours after injection, Phys. Med. Biol. 58, 3965 (2013).

[26] H. Paysen, N. Loewa, A. Stach, J. Wells, O. Kosch, S. Twamley, M. R. Makowski, T. Schaeffter, A. Ludwig, and F. Wiekhorst, Cellular uptake of magnetic nanoparticles imaged and quantified by magnetic particle imaging, Sci. Rep. 10, 1922 (2020).

[27] P. Vogel, M. A. Rückert, P. M. Jakob, and V. C. Behr, $\mu \mathrm{MPI}$ - initial experiments with an ultrahigh resolution MPI, IEEE Trans. Magn. 51, 7067522 (2015).

[28] S. A. Shah, R. M. Ferguson, and K. M. Krishnan, Slew-rate dependence of tracer magnetization response in magnetic particle imaging, J. Appl. Phys. 116, 163910 (2014).

[29] M. G. Christiansen, A. W. Senko, R. Chen, G. Romero, and P. Anikeeva, Magnetically multiplexed heating of single domain nanoparticles, Appl. Phys. Lett. 104, 213103 (2014).

[30] I. N. Weinberg, P. Y. Stepanov, S. T. Fricke, R. Probst, M. Urdaneta, D. Warnow, H. Sanders, S. C. Glidden, A. McMillan, P. M. Starewicz, and J. P. Reilly, Increasing the oscillation frequency of strong magnetic fields above $101 \mathrm{kHz}$ significantly raises peripheral nerve excitation thresholds, Med. Phys. 39, 2578 (2012).

[31] Z. W. Tay, D. Hensley, J. Ma, P. Chandrasekharan, B. Zheng, P. Goodwill, and S. Conolly, Pulsed excitation in magnetic particle imaging, IEEE Trans. Med. Imaging 38, 2389 (2019).

[32] H. Paysen, J. Wells, O. Kosch, U. Steinhoff, J. Franke, L. Trahms, T. Schaeffter, and F. Wiekhorst, Improved sensitivity and limit-of-detection using a receive-only coil in magnetic particle imaging, Phys. Med. Biol. 63, 13NT02 (2018).

[33] S. B. Trisnanto and Y. Takemura, Modulating relaxation responses of magnetic nanotracers for submillimeter imaging, Appl. Phys. Lett. 115, 123101 (2019).

[34] P. Guardia, A. Labarta, and X. Batlle, Tuning the size, the shape, and the magnetic properties of iron oxide nanoparticles, J. Phys. Chem. C 115, 390 (2011). 
[35] B. Pacakova, S. Kubickova, G. Salas, A. R. Mantlikova, M. Marciello, M. P. Morales, D. Niznansky, and J. Vejpravova, The internal structure of magnetic nanoparticles determines the magnetic response, Nanoscale 9, 5129 (2017).

[36] C. Shasha, E. Teeman, and K. M. Krishnan, Nanoparticle core size optimization for magnetic particle imaging, Biomed. Phys. Eng. Express 5, 055010 (2019).

[37] S. B. Trisnanto and Y. Kitamoto, Distributive activation volumes of magnetically interacting nanostructures, J. Phys. Chem. C 123, 23732 (2019).

[38] T. Kluth, P. Szwargulski, and T. Knopp, Towards accurate modeling of the multidimensional magnetic particle imaging physics, New J. Phys. 21, 103032 (2019).

[39] R. H. Kodama, Magnetic nanoparticles, J. Magn. Magn. Mater. 200, 359 (1999).

[40] D. V. Berkov, P. Görnert, N. Buske, C. Gansau, J. Mueller, M. Giersig, W. Neumann, and D. Su, New method for the determination of the particle magnetic moment distribution in a ferrofluid, J. Phys. D: Appl. Phys. 33, 331 (2000).

[41] P. Bender, C. Balceris, F. Ludwig, O. Posth, L. K. Bogart, W. Szczerba, A. Castro, L. Nilsson, R. Costo, H. Gavilán, D. González-Alonso, I. de Pedro, L. F. Barquín, and C. Johansson, Distribution functions of magnetic nanoparticles determined by a numerical inversion method, New J. Phys. 19, 073012 (2017).

[42] T. Yoshida, K. Enpuku, F. Ludwig, J. Dieckhoff, T. Wawrzik, A. Lak, and M. Schilling, in Magnetic Particle Imaging, edited by T. M. Buzug and J. Borgert (Springer, Berlin, Heidelberg, 2012), p. 3.

[43] S. B. Trisnanto, S. Ota, and Y. Takemura, Two-step relaxation process of colloidal magnetic nanoclusters under pulsed fields, Appl. Phys. Express 11, 075001 (2018).

[44] P. Ilg, Equilibrium magnetization and magnetization relaxation of multicore magnetic nanoparticles, Phys. Rev. B 95, 214427 (2017).

[45] Y. L. Raǐkher and M. I. Shliomis, in Advances in Chemical Physics, edited by W. Coffey (John Wiley \& Sons, Inc., New York, NY, 2007), p. 595.

[46] W. T. Coffey, P. J. Cregg, and Y. U. P. Kalmykov, in Advances in Chemical Physics, edited by I. Prigogine and S. A. Rice (John Wiley \& Sons, Inc., New York, NY, p. 263.

[47] R. M. Ferguson, K. R. Minard, A. P. Khandhar, and K. M. Krishnan, Optimizing magnetite nanoparticles for mass sensitivity in magnetic particle imaging, Med. Phys. 38, 1619 (2011).

[48] Z. W. Tay, D. W. Hensley, E. C. Vreeland, B. Zheng, and S. M. Conolly, The relaxation wall: Experimental limits to improving MPI spatial resolution by increasing nanoparticle core size, Biomed. Phy. Eng. Express 3, 035003 (2017).

[49] Q. Li, C. W. Kartikowati, S. Horie, T. Ogi, T. Iwaki, and K. Okuyama, Correlation between particle size/domain structure and magnetic properties of highly crystalline $\mathrm{Fe}_{3} \mathrm{O}_{4}$ nanoparticles, Sci. Rep. 7, 9894 (2017).

[50] J. Carrey, B. Mehdaoui, and M. Respaud, Simple models for dynamic hysteresis loop calculations of magnetic single-domain nanoparticles: Application to magnetic hyperthermia optimization, J. Appl. Phys. 109, 083921 (2011).

[51] J. Dieckhoff, D. Eberbeck, M. Schilling, and F. Ludwig, Magnetic-field dependence of Brownian and Néel relaxation times, J. Appl. Phys. 119, 043903 (2016).

[52] M. I. Shliomis, Ferrohydrodynamics: Testing a third magnetization equation, Phys. Rev. E 64, 060501(R) (2001).

[53] S. B. Trisnanto and Y. Kitamoto, Field-dependent Brownian relaxation dynamics of a superparamagnetic clusteredparticle suspension, Phys. Rev. E 90, 032306 (2014).

[54] F. Ludwig, C. Balceris, and C. Johansson, The anisotropy of the ac susceptibility of immobilized magnetic nanoparticles - the influence of intra-potential-well contribution on the ac susceptibility spectrum, IEEE Trans. Magn. 53, 7894242 (2017).

[55] S. Ota and Y. Takemura, Evaluation of easy-axis dynamics in a magnetic fluid by measurement and analysis of the magnetization curve in an alternating magnetic field, Appl. Phys. Express 10, 085001 (2017).

[56] H. Mamiya, Recent advances in understanding magnetic nanoparticles in ac magnetic fields and optimal design for targeted hyperthermia, J. Nanomater. 2013, 752973 (2013).

[57] M. Graeser, T. Knopp, P. Szwargulski, T. Friedrich, A. von Gladiss, M. Kaul, K. M. Krishnan, H. Ittrich, G. Adam, and T. M. Buzug, Towards picogram detection of superparamagnetic iron-oxide particles using a gradiometric receive coil, Sci. Rep. 7, 6872 (2017).

[58] S. B. Trisnanto and Y. Takemura, Complex magnetization harmonics of polydispersive magnetic nanoclusters, Nanomaterials 8, 424 (2018).

[59] T. Knopp and A. Weber, Sparse reconstruction of the magnetic particle imaging system matrix, IEEE Trans. Med. Imaging 32, 1473 (2013).

[60] A. Ozaslan, A. A. snd Alacaoglu, O. B. Demirel, T. Çukur, and E. U. Saritas, Fully automated gridding reconstruction for non-cartesian $\mathrm{x}$-space magnetic particle imaging, Phys. Med. Biol. 64, 165018 (2019).

[61] T. Sasayama, Y. Tsujita, M. Morishita, M. Muta, T. Yoshida, and K. Enpuku, Three-dimensional magnetic nanoparticle imaging using small field gradient and multiple pickup coils, J. Magn. Magn. Mater. 427, 144 (2017).

[62] S. A. Shah, D. B. Reeves, R. M. Ferguson, J. B. Weaver, and K. M. Krishnan, Mixed Brownian alignment and Néel rotations in superparamagnetic iron oxide nanoparticle suspensions driven by an ac field, Phys. Rev. B 92, 094438 (2015).

[63] L. R. Croft, P. W. Goodwill, and S. M. Conolly, Relaxation in X-space magnetic particle imaging, IEEE Trans. Med. Imaging 31, 2335 (2012).

[64] H. Bagheri and M. Hayden, Resolution enhancement in magnetic particle imaging via phase-weighting, J. Magn. Magn. Mater. 498, 166021 (2020).

[65] P. W. Goodwill and S. M. Conolly, Multidimensional xspace magnetic particle imaging, IEEE Trans. Med. Imaging 30, 1581 (2011). 